\title{
Mistica del Paradiso al limite del non rappresentabile. Par. XXX: word-painting dantesco e disegno Botticelliano - Analisi di un intercambio mediale*
}

\author{
Cornelia Klettke \\ Universität Potsdam \\ klettke@uni-potsdam.de
}

\begin{abstract}
Questo saggio si dedica al problema dell'intercambio mediale fra il word-painting dantesco e il disegno botticelliano. Sulla base di un minuzioso paragone del Canto XXX del Paradiso con il disegno botticelliano che lo illustra possiamo formulare primi approcci per un sistema di corrispondenze sviluppato da Botticelli per la sua trasposizione figurativa del testo poetico. L'analisi dei dettagli e degli arrangiamenti delle forme rappresentate rivela una sensibile compenetrazione di Botticelli nel mondo spiritual-religioso del Canto e nella sua poetica. Basandosi su dettagliate indicazioni contenute nel testo, l'artista traspone figurativamente con grande esattezza l'intenzione di un intero brano del Canto XXX (la prima visione: fiume di luce e «mirabil primavera», v. 61-78). Ciò facendo, egli sposta la metaforicità sovrannaturale dei versi danteschi attraverso una deviazione. Tale differenza intermediale viene realizzata attraverso la concretizzazione dell'espressione simbolica "faville vive» che Botticelli trasforma in angeli, in angeli avvolti in faville e nelle raggiere. L'ermetica del testo dantesco appare in questo caso trasposta nella ambivalenza tipica del 'quadro con segreto' o vexierbild consistente qui in un labirinto di forme che sortisce un effetto di mistero. La mistica del Paradiso, per la cui simbologia in Dante svolgono un ruolo straordinario le gemme con la loro metafisica della luce in Botticelli, che trasforma le gemme in creature angeliche, non viene rappresentata con la stessa sublimità del testo dantesco. Sebbene il pittore, maneggiando con virtuosismo la matita, sappia esprimere una preziosità nelle sue forme, questa forma di rappresentazione non è sufficiente a rendere la preziosità nel doppio senso in cui in Dante viene prodotta attraverso le gemme, la quale
\end{abstract}

* L'originale del presente articolo è apparso in tedesco col titolo Paradiesmystik im Grenzbereich des Nicht-Darstellbaren. Par. XXX: Dantes Wortgemälde und Botticellis Zeichnung - Analyse eines Medienwechsels, in Michèle Mattusch, Sylvia Setzkorn (a cura di), Imagination - Evokation - Bild: Reflexionsästhetische Dimensionen der Bild-Text-Beziehung in der italienischen Literatur, in "Letteratura \& Arte», Pisa-Roma, Fabrizio Serra Editore, 2010, pp. 55-83. Il testo è stato successivamente rivisto e modificato in vista della pubblicazione in lingua italiana. Per la sua eccellente traduzione sono grata a Roberto Ubbidente (Humboldt Universität zu Berlin). 
è da descrivere tanto allegoricamente quanto retoricamente. Tuttavia, grazie alla sottile ricezione della poesia dantesca e nonostante alcune differenze, il disegno botticelliano per il Canto XXX del Paradiso offre una trasposizione ben riuscita di una dimensione mistica della visione interiorizzata della trascendenza.

Parole chiavi: Intercambio intermediale fra il wordpainting dantesco e il disegno botticelliano (Par. XXX); Il sistema di corrispondenze testo-immagine; Esperienze luminose e visive; Il significato dell'allegoresi della pietra preziosa; Il problema della non rappresentabilità; Il fiume di luce, L'addio di Beatrice.

\begin{abstract}
This article addresses the problem of the medial shift between Dante's word paintings and Botticelli's drawings. A detailed comparison of Par. XXX with Botticelli's drawing for this canto is the basis for a first approach to a system of correspondences which Botticelli created for his pictorial transposition of the poetic text. The analysis of the details and arrangements of the depicted shapes reveals the degree of sensitiveness with which Botticelli delves into the religious and spiritual world of the canto as well as into its poetics. The artist transposes the intention of a whole section of Par. XXX (the first vision: flow of light and "mirabil primavera", v. 67-78) by referring closely to textual details. In the course of this, he displaces the supernatural imagery of the Dantean verses by means of an arbitrary divergence. This intermedial difference arises from putting the symbolic expression of "faville vive" (= living sparks), which Botticelli permutes into angels - angels with cloaks covered in flames and angels with halos - into form. Here, the hermeticism of Dante's text appears transposed into the ambigram of a seemingly mysterious labyrinth of shapes. In Dante's work, gemstones and their metaphysics of light play a prominent role in the symbolization of the mysticism of paradise. In Botticelli's permutation of the gemstones into angelic figures, this mysticism does not reflect the Dantean majesty. Although Botticelli is able to express a certain preciosity in his shapes by his masterly handling of his pencil, this presentation does not completely equal Dante's preciosity, which is created in a two-fold way by the gemstones and can be described both allegorically as well as rhetorically. Nevertheless and despite several differences, owing to the subtle reception of the Dantean poetics, Botticelli's drawing for Canto XXX of Paradiso presents an exceedingly well realized transposition of the mystic dimension of an internalized view of transcendence.
\end{abstract}

Keywords: The medial shift between Dante's word painting and Botticelli's drawing (Par. $\mathrm{XXX}$ ); A system of correspondences between text and picture; Light and visual experience; The meaning of the gemstone allegoresis; The problem of non-representability; The Flow of Light, Farewell to Beatrice. 


\section{L'iniziazione di Dante nelle sfere della trascendenza: esperienze mistiche della luce e del vedere}

Il modello di mondo alla base della concezione dantesca del Paradiso cristiano si avventura negli àmbiti della trascendenza. Trasgredendo i limiti, il Poeta cerca di comunicare con gli strumenti della poesia le apparizioni delle legioni celesti, dei Patriarchi, dei Beati, dei Santi, dei cori angelici, di Maria, Regina del Paradiso, su su fino alla Trinità. La 'visualizzazione' avviene con l'aiuto di magistrali effetti di luce basati in parte su esperienze ottiche della percezione della luce cosmica, in parte su impressioni del fuoco terrestre ma altresì su visioni risiedenti al di là della capacità percettiva dell'occhio umano. Il Paradiso cristiano di Dante costituisce l'astratto progetto di un ideale basato sui valori della Bellezza, della Verità e della Carità quale sublimazione dell'amore terreno secondo la concezione cristologica. Quest'universo, per l'uomo invisibile e irraggiungibile, è approcciato da Dante sulla scorta di metafore alla portata della facoltà immaginativa umana nonché di determinati processi ottici, specialmente di quello del riflesso.

\subsection{Beatrice nel suo significato allegorico: una superficie di 'proiezione' dell'ammirazione e dell'adorazione dell'anima del poeta in cerca del divino}

Il viaggio di iniziazione del poeta attraverso le sfere celesti del Paradiso è dominato dalla figura di Beatrice che fa parte della schiera dei Beati. Questo personaggio rappresenta una superficie su cui si proiettano l'ammirazione e l'adorazione dell'anima del poeta, tutta dedita alla ricerca del divino. Come in una camera d'eco, il nome di Beatrice risuona dalla Vita Nova nella Commedia. Nella Vita Nova la figura femminile appare quale finzione di Dante, con vaghe implicazioni autobiografiche, come protettrice dell'Amore, in parte dotata di tratti cristologici. Alcune allusioni fatte nella Commedia si lasciano intendere quali reminiscenze della Beatrice dell'opera poetica giovanile. Alla fine della Vita Nova (XLII) il poeta crea l'aggancio decisivo per la continuità del personaggio Beatrice:

[...] io spero di dicer di lei quello che mai non fue detto d'alcuna. E poi piaccia a colui che è sire de la cortesia, che la mia anima se ne possa gire a vedere la gloria de la sua donna, cioè di quella benedetta Beatrice, la quale gloriosamente mira ne la faccia di colui qui est per omnia secula benedictus ${ }^{1}$.

1. Cit. secondo l'edizione di Michele Barbi, La Vita Nuova di Dante Alighieri, Edizione critica, Firenze: Bemporad \& Figlio, 1932 (= Edizione Nazionale delle Opere di Dante, I); corsivo nel testo. Sull'annuncio del ritorno di Beatrice nella Vita Nova cfr. anche Corrado Bologna, Il Canto XXX del Paradiso, in Lectura Dantis Turicensis, III, Paradiso, a cura di Georges Güntert, Michelangelo Picone, Firenze: Franco Cesati Editore, 2002, pp. 457 472: 459. Cfr. inoltre Corrado Bologna, Il ritorno di Beatrice. Simmetrie dantesche tra Vita Nova, «petrose» e Commedia, Roma: Salerno Editore, 1998. 


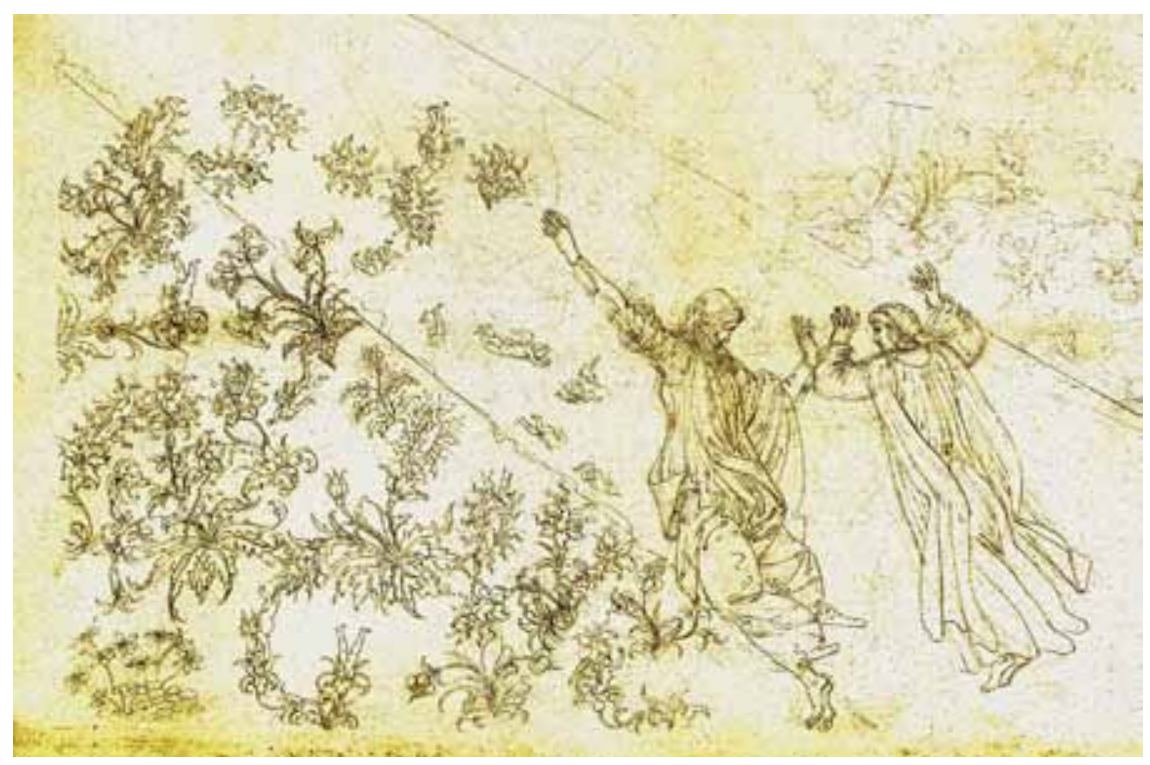

Figura 1. Sandro Botticelli (1444/45-1510), Paradiso XXX. Disegno facente parte del ciclo di illustrazioni della Divina Commedia di Dante. (Botticelli/Codex Hamilton 201).

${ }^{\odot}$ Kupferstichkabinett. Staatliche Museen zu Berlin.

Nella Divina Commedia Dante vede Beatrice nella sublimazione di un'apparizione celeste. In queste vesti essa assolve più funzioni. Nella terza Cantica essa rappresenta, oltre al protagonista, il principale agente, ossia la guida del viaggiatore umano in Cielo e allo stesso tempo il suo motore, come simbolo delle forze dinamiche dell'ascensione. Dal punto di vista allegorico Beatrice simboleggia la saggezza teologica come suprema virtù. Essa rende le verità di fede intelligibili al poeta, guidandolo, così, verso l'illuminazione interiore. Beatrice rappresenta Bellezza e Amore nella trascendenza. Da questo punto di vista essa prefigura e riflette la Vergine Maria. La sua presenza nel testo e la sua missione terminano con l'iniziazione del poeta nella Rosa dei Beati in cui Beatrice riprende il posto che aveva provvisoriamente lasciato per fare da guida a Dante (Par. XXX).

Per il protagonista la questione del femminile, nell'ambivalenza tra bellezza e amore terreni e celesti, si chiarisce già al culmine del Purgatorio, nel Paradiso terrestre, attraverso la prima epifania di Beatrice. Il personaggio di Dante perviene alla purificazione, ossia ad un'esperienza sublimata del femminile nell'incontro con Beatrice sullo sfondo del suo passato poetico (cfr. Vita Nova). Non è un caso che Beatrice compaia già nel Paradiso terrestre. Nel Dante pellegrino non ancora sottratto, nella sua virilità, alla dimensione terrena, la contemplazione di Beatrice nelle vesti di regina alla guida del trionfo allegorico della Chiesa (Purg. XXX) risveglia un ricordo che gli presentifica la cara immagine di un amore di gioventù. Il punto di partenza dell'incontro con il 
femminile è dunque costituito dall'esperienza sensoriale terrena. Attraverso la sua bellezza sovrannaturale, la beata Beatrice guida il desiderio dell'uomo nelle sfere della pura spiritualizzazione / intimizzazione. All'uomo (alla persona umana) peccatore essa richiede di conoscere se stesso, provocando l'ammissione di colpa di Dante e il pentimento per le debolezze della carne e per l'infedeltà (Purg. XXXI). Solo dopo la morte simbolica del vecchio Adamo l'uomo può contemplare i misteri della Fede.

\subsection{Esperienze luminose e visive: il sistema dei (retro)riflessi}

Per poter ammirare da vicino l'apparizione sovrannaturale di Beatrice, il desideroso e anelante pellegrino deve varcare il limite rappresentato dal fiume Letè. Solo dopo uno svenimento, quale perdita della coscienza e morte simbolica, nonché dopo ripetute immersioni nell'acqua del fiume, quale atto di purificazione e dimenticanza, il poeta viene reso partecipe dell'esperienza mistica che consiste nel poter guardare il volto senza veli di Beatrice. Il ricordo dell'amore giovanile è impallidito al cospetto della luce soprannaturale del sapere divino emanata dal volto femminile. Lo svelamento degli occhi costituisce un'esperienza cruciale sulla via del poeta verso la percezione e l'esperienza del divino nella trascendenza:

Così cantando ${ }^{2}$ cominciaro; e poi

al petto del grifon seco menarmi, ove Beatrice stava volta a noi.

Disser: «Fa che le viste non risparmi;

posto t'avem dinanzi a li smeraldi

ond' Amor già ti trasse le sue armi». ${ }^{2}$

Mille disiri più che fiamma caldi

strinsermi li occhi a li occhi rilucenti,

che pur sopra 'l grifone stavan saldi.

Come in lo specchio il sol, non altrimenti

la doppia fiera dentro vi raggiava,

or con altri, or con altri reggimenti. (Purg. XXXI, 112-123) ${ }^{3}$

In questi versi il poeta Dante inscena la sua prima esperienza trascendente del Celeste ancor prima del suo ingresso in Paradiso. Tale esperienza è basata su un vedere affatto interiorizzato. Per poter rappresentare ciò, il poeta ricorre a una serie di immagini e simboli nonché al sapere alchemico medievale circa le pietre preziose. Negli occhi verde smeraldo ${ }^{3}$ di Beatrice si riflette il simulacro

2. Si tratta delle quattro figure femminili che accompagnano il carro trionfale sulla sinistra e che generalmente vengono interpretate come allegorie delle quattro virtù cardinali.

3. Nel Medio Evo lo smeraldo veniva usato come specchio. Cfr. Dante Alighieri, Commedia con il commento di Anna Maria Chiavacci Leonardi, Bologna: Zanichelli, 2001, commento a Purg. XXXI, verso 116, p. 568. Cfr. anche Dante Alighieri, Die göttliche Komödie. Erläutert von Ferdinand Barth aufgrund der Übersetzung von Walter Naumann, Darmstadt: Wissenschaftliche Buchgesellschaft, 2004, pp. 340-341. Cfr. Valeria Bertolucci 
di una figura d'animale, il grifone, come simbolo di Cristo. Questa figura traina il carro del trionfo su cui troneggia Beatrice come allegoria della Teologia. Dante vede il Figlio di Dio in una molteplice sottrazione:

- non in forma umana ma nella sua manifestazione allegorica come grifone (doppia natura Dio-Uomo)

- quest'immagine dell'essere fiabesco solo indirettamente come immagine riflessa negli occhi di Beatrice

- l'illusoria immagine riflessa del grifone oscillante nella sua 'doppia natura' tra leone e aquila mentre emana raggi sovrannaturali

- Beatrice come allegoria delle verità di Fede che riflette i raggi

Il paragone del Grifone con il sole è un raddoppiamento del rimando a Cristo. Questo complesso sistema di (retro)riflessi, in cui Dante percepisce solo il riverbero di un'allegoria di Cristo, inscena l'enigmatizzazione ottenuta con l'aiuto (dell'energia) della luce.

\subsection{Il significato dell'allegoresi della pietra preziosa per l'interazione degli sguardi: la forza degli occhi di smeraldo}

Tali raggi vengono irradiati dagli occhi di Beatrice paragonati agli smeraldi, un metaforismo che va visto più da vicino. Il significato degli occhi come smeraldi risulta riconoscibile già dall'uso sineddotico «Merrenti a li occhi suoi; [...]» (Purg. XXXI, 109) e "posto t'avem dinanzi a li smeraldi» (Purg. XXXI, 116). Le pietre simboleggiano il personaggio di Beatrice e dominano da qui in avanti la comunicazione tra i personaggi. Inoltre, in Dante lo smeraldo quale attributo di bellezza muliebre ${ }^{4}$ possiede altre connotazioni che svolgono

Pizzorusso, Gli smeraldi di Beatrice (1969), in ID, Morfologie del testo medievale, Bologna: Il Mulino, 1989, pp. 199-207: 206. Per un approfondimento del motivo degli occhi di Beatrice come smeraldi cfr. infra.

4. Per la concezione degli occhi di smeraldo quale attributo di bellezza femminile la fonte di Dante è costituita dal Tresor (III, 13. 11) del suo maestro Brunetto Latini, il quale a sua volta riproduce un passo tratto dalla descrizione della bellezza femminile fatta da Geoffroi de Vinsauf nella sua Poetria nova. Cfr. Bertolucci Pizzorusso, op. cit., pp. 204-205, che attribuisce un significato affatto particolare all'influenza di Latini sul sistema allegorico dantesco delle pietre preziose. Su ciò cfr. il discorso di Bertolucci su A. Levavasseur [«Les pierres précieuses dans la Divine Comédie», in Revue des Études italiennes, 4, 1957, pp. 31-100] riguardo a Marbod [Bertolucci Pizzorusso, op. cit., pp. 200-203]. Nel Tresor, III, 13. 11 Latini fa riferimento alla descrizione di Iseult secondo la quale i suoi occhi sono più belli degli smeraldi: "Autresi fist Tristans, quant il devisa la biauté ma dame Isseut: 'Ses cheviaus, fist il, resplandis[se]nt come fil d'or; son front sormonte la flor de lis; ses noirs sorcils sont ploié come petis arconciaus; une petite voie de l[eit] le desevre par mi la ligne dou nes, [et] est si par mesure qu'il n’i a ne plus ne moins; ses iauz qui sormontent toutes esmeraudes reluissent en son front come .ii. estoilles [...]' ». [Cit. secondo l'edizione del Tresor, a cura di Pietro G. Beltrami, Torino: Einaudi, 2007, p. 666]. In Dante, invece, gli occhi sono fatti di pietre preziose, meglio ancora: gli smeraldi vengono trasformati in una sineddoche di Beatrice attraverso cui essa (Beatrice) viene elevata nella sfera del soprannaturale. In questo caso l'uso sineddotico 


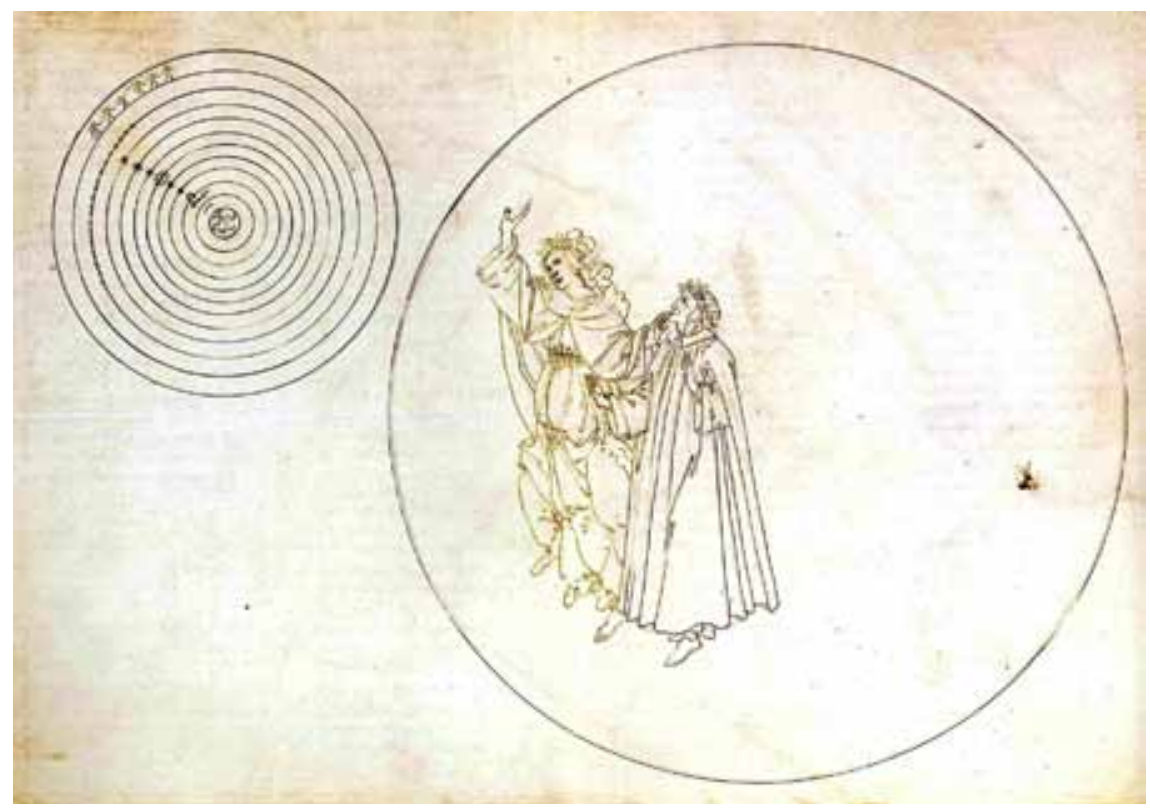

Figura 2. Sandro Botticelli (1444/45-1510), Paradiso II. Disegno facente parte del ciclo di illustrazioni della Divina Commedia di Dante. (Botticelli/Codex Hamilton 201).

${ }^{\circledR}$ Kupferstichkabinett. Staatliche Museen zu Berlin.

un ruolo importante per la comprensione del personaggio Beatrice nella sua interazione con il pellegrino Dante. A prescindere dall'associazione con l'eterna primavera che accompagna la bellezza, questa gemma è anche simbolo di Giustizia ${ }^{5}$. Tale virtù è riferibile al sermone di Beatrice che precede lo svelamento dei suoi occhi. Il suo sguardo è talmente penetrante da sortire effetto anche attraverso il velo: «drizzar li occhi ver' me di qua dal rio.» (Purg. XXX, 66). Dinanzi all'incorruttibile sguardo di smeraldo non è possibile alcuna via di fuga, nessuna simulazione o dissimulazione. L'intransigenza del simbolismo attribuito allo smeraldo contiene in sé anche la castità ${ }^{6}$ quale presup-

della parola smeraldo rinvia sin d'ora alla successiva sospensione delle leggi naturali (eterno al posto di effimero; cfr. infra, paragrafo 3.1).

5. Brunetto Latini attribuisce lo smeraldo alla virtù della iustitia: « La quarte vertu est justise, qui est senefiee por l'esmeraude, qui est la plus usee vertus et la plus belle chose que oil d'ome peust veoir» [Tresor (II, 1. 3), cit. da Beltrami, op. cit., pp. 330-333]. Cfr. anche Bertolucci Pizzorusso, op. cit., pp. 205-206; cfr. ancora Albertus Magnus in De mineralibus (II, XVII, voce "Smaragdus») sul rafforzamento delle capacità retoriche attraverso lo smeraldo.

6. Cfr. Albertus Magnus [De mineralibus, II, XVII, voce «Smaragdus», pp. 44-45], che mette in rilievo il fatto che lo smeraldo inciti chi lo porta alla castità. Cfr. anche Eugenio CHIARINI (a cura di), Il canto XXX del Purgatorio, in Lectura Dantis Scaligera, Firenze: Le Monnier, 1965, pp. 1103-1138: 1108. 
posto per la purificazione. Inoltre, allo smeraldo vengono attribuiti anche poteri curativi per gli occhi ${ }^{7}$. A questa capacità degli occhi di Beatrice si allude in Paradiso XXVI:

perché la donna che per questa dia region ti conduce, ha ne lo sguardo la virtù ch'ebbe la man d'Anania. (Par. XXVI, 10-12)

Il potere curativo degli occhi di smeraldo di Beatrice si rivela un motivo ricorrente dopo ogni accecamento che il viaggiatore Dante sperimenta nelle epifanie che egli incontra. A questa capacità (virtus) si collega anche la qualità che consiste nel trasmettere energia soprannaturale al suo protetto. La brillantezza degli occhi - lo splendore degli smeraldi - dà impulsi che sopraffanno e rafforzano il pellegrino in Cielo. Ricorrendo al simbolismo dello smeraldo, l'Autore rappresenta la sua forza di resistere allo sforzo sovrumano conferitagli dalla trasmissione di energia operata dagli occhi di Beatrice. Nelle più diverse variazioni il testo ricorre a questo fenomeno unico dei poteri di irradiare e brillare di Beatrice, la cui portata ottica misura dimensioni inimmaginabili.

così de li occhi miei ogne quisquilia

fugò Beatrice col raggio d'i suoi,

che rifulgea da più di mille milia: (Par. XXVI, 76-78)

L'ascesa di Dante nelle sfere celesti e il suo percorso attraverso il Paradiso sono profondamente caratterizzati da ammirazione e stupore. La bellezza soprannaturale della guida accende l'entusiasmo del poeta, i suoi occhi scintillanti trasmettono impulsi di energia all'anelante pellegrino. Il mistero della visione che egli sperimenta successivamente gli viene rivelato attraverso un contatto visivo, attraverso un linguaggio degli occhi che, a mo' di leitmotiv, influenza e determina gli stadi della sua ascesa immaginaria, ovverossia del suo contemplare. La comunicazione degli sguardi diviene così l'essenza dell'interazione tra Dante e Beatrice. La rappresentazione dell'esperienza luminosa nel (retro)riflesso si rivela essere un ulteriore privilegiato processo testuale poetico con cui Dante mette in scena la sua esperienza della trascendenza nell'aldilà del Paradiso.

\section{Paradiso XXX. L'Empireo - un Utopos. L'immaginabile terrestre - una metafora}

La nostra analisi tralascia il percorso attraverso le sfere celesti per rivolgersi direttamente al Canto XXX del Paradiso in cui Dante, accompagnato da Beatrice e proveniente dal cielo cristallino o Primo Mobile, si dirige verso l'Empi-

7. Cfr. Bertolucci Pizzorusso, op. cit., pp. 205-206.

8. Cfr. Bertolucci Pizzorusso, op. cit., pp. 205-206. L'accecamento di Saulo nell'epifania di Cristo viene guarita con l'imposizione delle mani. Dante imita in questo punto in una riscrittura la trasformazione di Saulo in Paolo narrata negli Atti degli Apostoli 9, 7-19. 
reo, il supremo cerchio della trascendenza in cui risiedono la Candida Rosa e la Trinità. Questo passo da un Cielo all'altro è pensato come passaggio dalla più grande sfera materiale alla sfera dell'immateriale ${ }^{9}$, dello spirito, che secondo la descrizione fattane da Beatrice è "pura luce»:

Noi siamo usciti fore

del maggior corpo al ciel ch'è pura luce:

luce intellettüal, piena d'amore;

amor di vero ben, pien di letizia; (Par. XXX, 38-41)

Nella sua forma terrena di uomo il pellegrino Dante non può raggiungere questa sfera con il suo corpo, bensì solo con lo spirito e l'anima. Pertanto il testo non offre punti di appoggio per un prosieguo del movimento fisico nel senso di un'ulteriore ascensione come nelle sfere precedenti. A partire dall'inizio del Canto XXX del Paradiso non è infatti più riconoscibile alcuna continuazione del movimento. La corporeità diviene una metafora, laddove il corpo appare per così dire come fantasma. Si tratta di un muoversi e un toccare dell'anima. L'immersione del poeta nell'allegoria della rosa celeste come massima esperienza trascendente si comprende come esperienza mistica dell'anima. Da questo punto di vista l'Empireo rappresenta un Utopos ${ }^{10}$ che si sottrae ad una collocazione topografica in base alle leggi della fisica e della matematica. Di conseguenza, nel testo non è riconoscibile alcuna indicazione di luogo riferita ad un 'ingresso' in questa sfera né tanto meno il momento preciso dello stesso.

\subsection{Luce e sentimento: la mutata percezione di Dante - dal «nulla vedere» alla "novella vista»}

Il cielo immobile dell'Empireo simboleggia il venir meno delle categorie di spazio e tempo e con ciò l'eternità come la santità della pienezza di purissima luce eterna e incommensurabile nella fusione con il sentimento come più grande amore e suprema gioia. Già in precedenza il pellegrino Dante ha potuto gettare uno sguardo dal cielo cristallino all'Empireo e, abbacinato, ha chiuso gli occhi (Par. XXVIII, 16-18) ${ }^{11}$. In occasione della sconvolgente pienezza di luce che gli si presenta con l'Empireo egli si ricorda di questa esperienza di luce con una retorica ermetica che rinvia alla 'sovraspazialità'

9. Andreas Kablitz (Poesie der Wissenschaft: Dantes Kosmologie, in Herbert Jaumann, Jürgen Klein, Bettina Rommel, Gregor Vogt-Spira (a cura di), Domänen der Literaturwissenschaft, Tübingen: Stauffenburg, 2001, pp. 233-252: 235) fa notare che l'Empireo di Dante si differenzia dal precedente modello cristiano-cosmologico poiché rappresenta una sfera «incorporale».

10. Corrado Bologna (Il Canto XXX del Paradiso, op. cit., p. 464; corsivo nel testo) rinvia alla definizione dell'anima in Agostino: «luogo utòpico, luogo senza luogo, locus qui non est locus...".

11. «un punto vidi che raggiava lume / acuto sì, che 'l viso ch'elli affoca / chiuder conviensi per lo forte acume». 
del divino. Questa sospensione del contrasto tra dentro e fuori a favore dell'illimitatezza non è afferrabile in un'immagine ma resta inimmaginabile per lo spirito umano.

Non altrimenti il trïunfo che lude sempre dintorno al punto che mi vinse, parendo inchiuso da quel ch'elli 'nchiude, (Par. XXX, 10-12)

La descrizione dell'Empireo comporta per il poeta dei problemi fin'allora sconosciuti. L'abbacinamento provocato dalla quantità di luce evoca il «nulla vedere» (v. 14), ovvero la negazione del vedere a cui si accompagna l'incapacità di verbalizzare di chi scrive. È il fallimento della facoltà visiva terrena. L'uomo non è in grado di guardare il cielo divino. Anche la bellezza di Beatrice acquista un potere irradiante talmente schiacciante da superare la capacità intellettiva umana tanto che il poeta è costretto a dichiararsi vinto. Beatrice rinvia all'immaterialità dell'Empireo che è incorporeo, fatto di solo spirito e sentimento; qualità, questa, simboleggiata dalla metafora della luce (Par. XXX, 39-40, cfr. supra). Con l'aiuto di Beatrice, in un'azione spettacolare, l'anelante pellegrino perviene ad un livello superiore del vedere / del guardare. Egli si sente circondato a pieno dalla luce e avvolto in un velo di splendore luminoso, tanto da esserne completamente abbacinato:

così mi circunfulse luce viva, e lasciommi fasciato di tal velo del suo fulgor, che nulla m’appariva. (Par. XXX, 49-51)

Per il poeta quest'esperienza mistica comporta una radicale trasformazione:

Non fur più tosto dentro a me venute queste parole brievi, ch'io compresi me sormontar di sopr' a mia virtute; e di novella vista mi raccesi tale, che nulla luce è tanto mera, che li occhi miei non si fosser difesi; (Par. XXX, 55-60)

Il personaggio Dante viene dotato di poteri e capacità percettive sovrannaturali. Egli supera i suoi limiti, pervenendo ad un nuovo tipo di vedere («novella vista», v. 58), che lo rende capace di ammirare l'allegoricità e la metaforicità dell'immateriale, ossia l'Empireo. Il nuovo sguardo è diretto sulla trasformazione di materialità in immaterialità, di temporalità in eternità, che la lingua di Dante può esprimere solo attraverso metafore e paragoni. Con l'acquisto della nuova capacità percettiva narrata fino al verso 60 termina la prima parte del Canto XXX del Paradiso. 


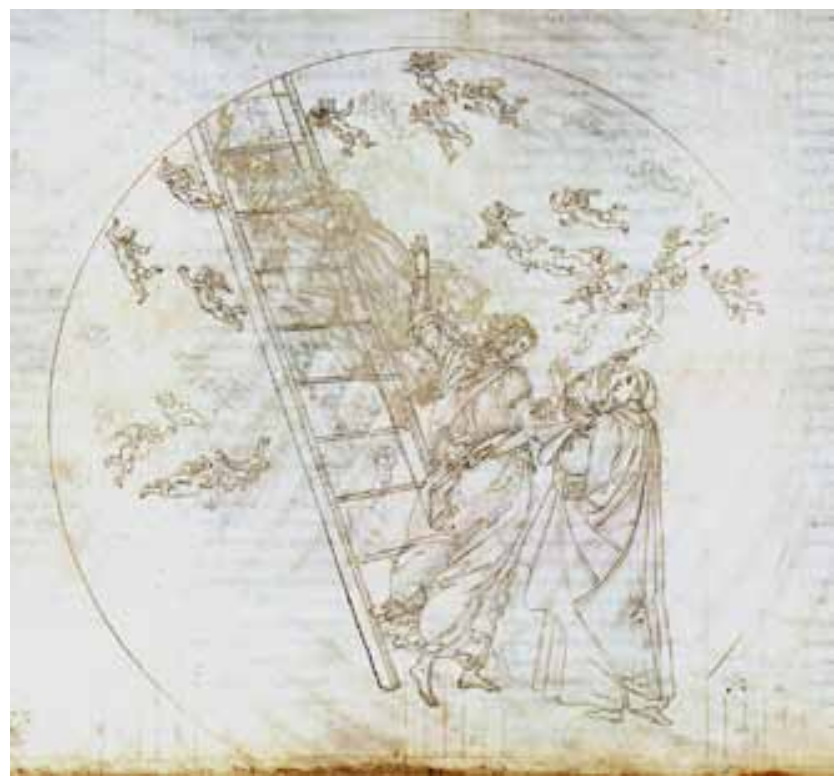

Figura 3. Sandro Botticelli (1444/45-1510), Paradiso XXI. Disegno facente parte del ciclo di illustrazioni della Divina Commedia di Dante. (Botticelli/Codex Hamilton 201).

${ }^{\odot}$ Kupferstichkabinett. Staatliche Museen zu Berlin.

\subsection{La prima visione dantesca dell'Empireo: il fiume di luce e la "mirabil primavera"}

La seconda parte del Canto contiene la prima visione dell'Empireo da parte di Dante (fino al verso 96) come cuore dell'intero Canto e come preliminare dell'ingresso alla Rosa dei Beati, che rappresenta la terza parte del Canto. L'Illuminazione interiore della "novella vista» (v. 58) pone il visitatore dell'Aldilà nella condizione di percepire il meraviglioso panorama del fiume luminoso nell'Empireo che gli appare direttamente come una visione.

e vidi lume in forma di rivera fulvido di fulgore, intra due rive

63 dipinte di mirabil primavera.

Di tal fiumana uscian faville vive, e d'ogne parte si mettien ne'fiori,

66 quasi rubin che oro circunscrive; poi, come inebrïate da li odori, riprofondavan sé nel miro gurge,

69 e s'una intrava, un'altra n'uscia fori.

«L'alto disio che mo t'infiamma e urge, d'aver notizia di ciò che tu vei,

72 tanto mi piace più quanto più turge; 
ma di quest' acqua convien che tu bei prima che tanta sete in te si sazi»:

75 così mi disse il sol de li occhi miei.

Anche soggiunse: "Il fiume e li topazi ch'entrano ed escono e 'l rider de l'erbe

78 son di lor vero umbriferi prefazi.

Non che da sé sian queste cose acerbe; ma è difetto da la parte tua,

81 che non hai viste ancor tanto superbe».

Non è fantin che sì sùbito rua col voto verso il latte, se si svegli

84 molto tardato da l'usanza sua, come fec' io, per far migliori spegli ancor de li occhi, chinandomi a l'onda

87 che si deriva perché vi s'immegli; e sì come di lei bevve la gronda de le palpebre mie, così mi parve

90 di sua lunghezza divenuta tonda.

Poi, come gente stata sotto larve, che pare altro che prima, se si sveste

93 la sembianza non süa in che disparve, così mi si cambiaro in maggior feste li fiori e le faville, sì ch'io vidi

96 ambo le corti del ciel manifeste. (Par. XXX, 61-96)

\subsubsection{La prefigurazione della Rosa dei Beati}

Questa prima visione dell'Empireo (Par. XXX, 61-96) riflette già il Paradiso celeste così come si svelerà più avanti con la Candida Rosa. Con l'aiuto di Beatrice, gli occhi di Dante, desiderosi di ammirare, sono stati già sensibilizzati dalla precedente esperienza luminosa che li ha preparati a ricevere una percezione della natura divina (Par. XXX, 57-60). La facoltà visiva del poeta, tuttavia, non è ancora così perfetta da poter riconoscere la seconda grande visione dell'Empireo, ossia la Rosa dei Beati. La vera e propria immagine perfetta e senza veli, che fa apparire gli angeli e i Beati come figure, è preceduta da una prefigurazione, la rappresentazione del fiume di luce, che sotto forma di metafora vela l'ultimo mistero. Per esprimere il celeste Dante si serve di paragoni terreni. Questo straniante velamento cala come una cortina dinanzi alla rivelazione. Su tale cortina è dipinto un panorama («dipinte di mirabil primavera», v. 63) che si mostra adeguato alla facoltà immaginativa umana. Attraverso la magnificenza dei fiori primaverili sulle 'rive' del fiume di luce si innesca una lontana reminiscenza della tradizione del locus amoenus e della poesia pastorale antica di un Virgilio. L'artificiosità di questo word-painting e la sua retorica appaiono come una mise en abyme di una sequenza nel Paradiso terrestre (Purg. XXVIII, 25-69) in cui si dispiega un paesaggio bucolico con tanto di evocazione del mito di Proserpina (Ovid, Met. V, 385 ss). Tale brano suggerisce il legame della fertilità con la purezza sullo sfondo del metaforismo dei fiori e, similmente ai versi riguardanti il fiume di luce, anticipa l'inizio di 
Par. XXXI, 1-21, che con la rappresentazione poetica della Candida Rosa sembra inscenare una ripetizione differente del metaforismo floreale.

\subsubsection{Evidenze semantiche e ambivalenze sulla spiritualizzazione dell'immagine} Con la descrizione del fiume di luce (Par. XXX, 61-96) il poeta innalza il suo testo a un livello 'superiore' che deve rappresentare una trasgressione nella sfera del metafisico e del divino. Recentemente Anna Maria Chiavacci Leonardi ha fatto notare l'uso di rari latinismi («miro gurge», v. 68; «turge», v. 72) in grado di suggerire una spiritualizzazione dell'immagine che viene ad essere non più di questo mondo ${ }^{12}$. Inoltre il poeta ricorre a ulteriori strumenti poetici. Già per questa trasfigurazione di un fiume terreno guadagna valenza la sua tardiva spiegazione: «ché dove Dio sanza mezzo governa, / la legge natural nulla rileva» (Par. XXX, 122-123) ${ }^{13}$. In tal modo viene sospeso il contrasto basilare di acqua e fuoco: «lume», «rivera» (v. 61); «rive» (v. 62); «Di tal fiumana uscian faville» (v. 64); «gurge» (v. 68); «acqua» (v. 73); «Il fiume» (v. 76) ${ }^{14}$. L'ambivalenza della semantica oscillante tra acqua e fuoco suggerisce l'onnipotenza trasgressiva del divino sulla materia. Il metaforismo di questo brano imita questa fusione dello scorrere e del bruciare. Allo stesso tempo si crea un legame tra il fiume di luce del Paradiso celeste e il fiume Letè del Paradiso terrestre.

\subsubsection{L'allegoresi della gemma: rubino e topazio per la poetizzazione e la produzione degli "umbriferi prefazi»}

Questo metaforismo viene arricchito attraverso un ulteriore accorgimento poetico - il simbolismo delle pietre preziose - che allo stesso tempo rappresenta un simbolismo cromatico nel senso dell'illusione creata di un dipinto. Il rosso del rubino (v. 66) e il giallo del topazio (v. 76) creano l'idea cromatico-sensibile di scintille («faville vive», v. 64). I versi di Par. XXX, 64 e seguenti, che

12. Cfr. l'edizione testuale di Chiavacci Leonardi, op. cit., commento a Par. XXX, 68, p. 544.

13. Kablitz (op. cit., pp. 242 ss.) spiega la sospensione delle leggi naturali per quanto riguarda i versi Par. XXX, 97-123.

14. Opinioni dissenzienti si registrano sulla prima parola del verso 62: «fulvido» o «fluvido». Sulla lettura di "fluvido» (dal lat. fluvidus) cfr. Natalino SAPEgno [nel commento a Par. XXX, 62, in Dante Alighieri, La Divina Commedia, a cura di N. Sapegno, III: Paradiso, Firenze: La Nuova Italia, $1982^{14}\left(1957^{1}\right)$, pp. 380-381], che in ciò segue l'edizione della Commedia curata da G. Vandelli (Milano, 1928). La soluzione «fluvido» è anche proposta da Walter Binni [Il canto XXX del Paradiso (1965), in Lectura Dantis Scaligera, III: Paradiso, Firenze: Le Monnier, 1968, pp. 1061-1092: 1075]. Cfr. inoltre la traduzione tedesca del passo ad opera di Hermann Gmelin. La ricerca attuale, invece, rifacendosi a Petrocchi, preferisce «fulvido» (dal lat. fulvidus, derivato di fulvus). Chiavacci Leonardi (op. cit., commento aggiuntivo al verso 62, p. 552) ricorda che il lat. fulvidus è documentato come designazione del colore dell'oro (cfr. «metallum fulvidum»). - «Fulvido» (color oro, rosso oro) prefigura il verso "quasi rubin che oro circunscrive» (Par. XXX, 66). Allo stesso tempo, in «fulvido" riecheggiano i fiori del Paradiso terrestre (Purg. XXVIII, 55: "volsesi in su i vermigli e in su i gialli / fioretti verso me») e con ciò una reminiscenza stilnovistica di Guinizzelli («tutti color di fior', giano e vermiglio» [Rime X, 6]). Cfr. Poeti del Duecento, a cura di Gianfranco Contini, Milano-Napoli: Riccardo Ricciardi Editore, 1960, tomo II, p. 472. 
preformano l'immagine degli angeli che salgono e scendono e che sprofondano nell'interno della Rosa mistica (Par. XXXI, 4-21), consentono di interpretare le «faville» come prefigurazioni degli angeli e i fiori, in cui si inseriscono le scintille degli angeli, come prefigurazioni dei Beati. Il rosso rubino delle scintille viene incorniciato dall'oro dei fiori ${ }^{15}$. Con la sua retorica, nel verso «quasi rubin che oro circunscrive» il poeta fa apparire, come per magia, una gemma preziosa dinanzi agli occhi del lettore, stimolandone la fantasia e indirizzandola sull'unicità, la eccezionalità e la inestimabilità della sua visione, la quale è anche un'esperienza di luce. I fiori di gemme come immagine dei Beati non connotano l'appassimento e quindi l'effimero. Come "perpetüi fiori» essi simboleggiano «l'etterna letizia» (Par. XIX, 22-23) e l'eterno amore che riempie di sé l'Empireo («ciel ch'è pura luce: / luce intellettüal, piena d'amore», Par. XXX, 39-40), l'amore che tutto ordina e tutto muove. Dal momento che i raggi del sole si infrangono nei rubini, essi irradiano uno splendore sovrannaturale. A questo fenomeno di particolare forza luminosa lo stesso Dante rinvia in un altro testo:

[...] certi corpi, per molta chiaritade di diafano avere in sé mista, tosto che 'I sole li vede, diventano tanto luminosi, che per multiplicamento di luce in quelli è lo loro aspetto [vincente], e rendono alli altri di sé grande splendore, sì come è l'oro e alcuna pietra ${ }^{16}$.

La questione del carattere simbolico dei rubini che chiama in causa l'alchimia medievale, è contemplata dalla nostra interpretazione dal momento che i segnali testuali legati al rubino, al topazio e allo smeraldo suggeriscono la pressante ipotesi che Dante fosse iniziato a questo sapere alchemico circa le proprietà delle pietre preziose. Com'è noto, il «carboncle» (it. carbonchio) è l'antico nome del rubino, pietra che riluce di un rosso vivo. La sua luminosità era proverbiale: «Par trestut ou ele est, si done lumere» ${ }^{17}$. L'etimologia risale probabilmente al colore rosso del carbone ardente (cfr. gr. anthrax): «Carbuncle veint tutes ardauntes pieres, kar ele getet rais de tutes parz cume charbun vif, et pur iceo ad num charbuncle» ${ }^{18}$. All'inizio del secondo Libro del suo Tresor Brunetto Latini discetta sul sistema delle gemme preziose nell'insegnamento dei dotti in merito al valore delle pietre per la vita degli uomini per quanto riguarda la bellezza, il piacere e la virtù. Rubini, zaffiri, diamanti e smeraldi: a ciascuna di queste pietre viene attribuita una virtù terrena. Abbiamo già ricor-

15. Cfr. l'edizione di SApegno, op. cit., commento a Par. XXX, 66, p. 381 . Il verso virgiliano (Aen. X, 134), puntualmente citato dai commenti a Par. XXX, 66, non contiene esplicitamente il termine 'rubino', bensì quello di pietra preziosa («gemma»).

16. Dante Alighieri, Convivio, III, VII 3; cit. secondo l'Edizione Nazionale delle Opere di Dante, a cura di Franca Brambilla, Firenze: Le Lettere, 1995, p. 189.

17. Paul Studer, Joan Evans, Anglo-Norman Lapidaries, Paris: Champion, 1924, p. 153, VIII. 9-10; cfr. anche Adolf Tobler, Erhard Lommatzsch, Altfranzösisches Wörterbuch, Berlin: Weidmann e Wiesbaden, Steiner, 1915 sgg., II, voce «charbocle», p. 256.

18. Studer, Evans, Anglo-Norman Lapidaries, op. cit., p. 110, LI; cfr. anche Tobler, LomMATZSCH, op. cit., p. 256. 


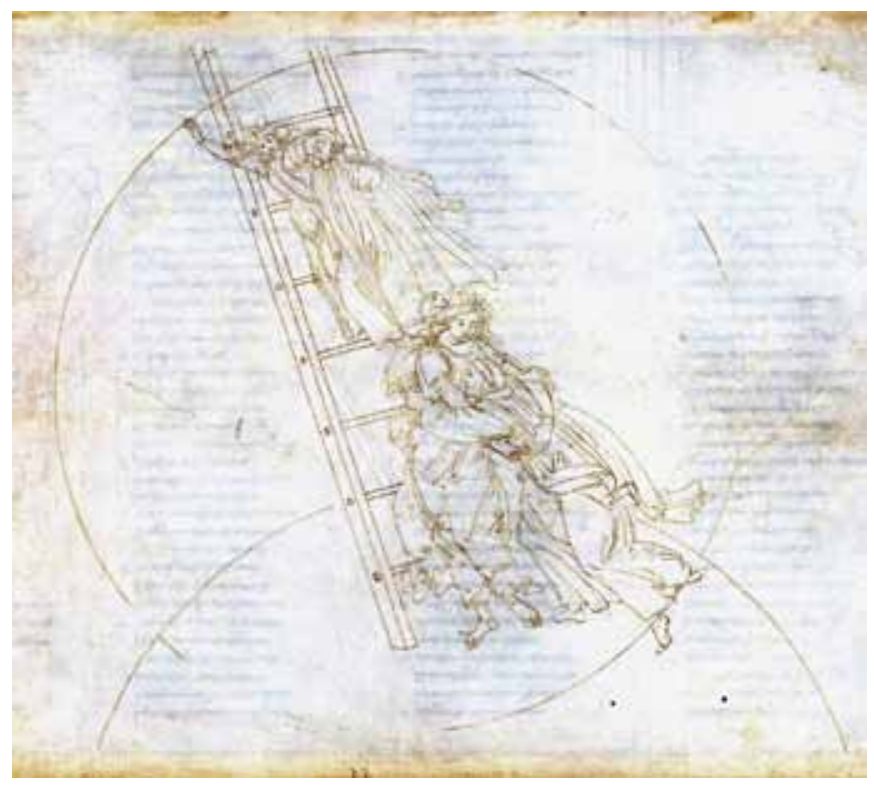

Figura 4. Sandro Botticelli (1444/45-1510), Paradiso XXII. Disegno facente parte del ciclo di illustrazioni della Divina Commedia di Dante. (Botticelli/Codex Hamilton 201).

${ }^{\odot}$ Kupferstichkabinett. Staatliche Museen zu Berlin.

dato la connotazione dello smeraldo con la Iustitia $^{19}$. Sul rubino, Latini osserva: «Et cest enseignement sera sor les .iiii. vertus a[ctiv]es, dont la premiere est prudence, qui est senefiee por le carboncle qui alumine la nuit et resplendist sor toutes pierres» ${ }^{20}$.

Oltre all'evocazione del rubino in Paradiso XXX, 66, vi è in tutta la Commedia solo un altro riferimento a questa gemma: "parea ciascuna rubinetto in cui / raggio di sole ardesse sì acceso, / che ne' miei occhi rifrangesse lui» (Par. $\mathrm{XIX}, 4-6)$. In questo caso la pietra splendente di rosso fuoco funge da immagine dei redenti che appaiono nel Cielo di Giove come epifania di un'aquila tempestata di rubini. I rubini accesi dal sole connotano anche il calore emanato da molti carboni ardenti come tante fiamme d'amore ("Così un sol calor di molte brage / si fa sentir, come di molti amori», Par. XIX, 19-20).

Nella fitta trama del tessuto del testo poetico dei versi Par. XXX, 66-70 la radiosa luminosità del rosso rubino entra in riferimento semantico col verso "L'alto disio che mo t'infiamma e urge». Il desiderio nei confronti dell'Altissimo («[l]'alto disio») si rafforza attraverso il verbo («infiamma») che a sua volta rinvia alle scintille color rosso rubino. Con ciò si ha uno spostamento del rosso rubino sul cercatore della Verità il cui acceso desiderio è ormai arrivato al punto

19. Cfr. supra, paragrafo 1.3 .

20. Latini, Tresor, II, 1.3; cit. da Beltrami, op. cit., pp. 330-331. 
massimo di tensione («tanto mi piace più quanto più turge»). Dal punto di vista di Beatrice, i rubini vengono sostituiti nel suo discorso con i topazi («Il fiume e li topazi / ch'entrano ed escono e 'l rider de l'erbe / son di lor vero umbriferi prefazi», Par. XXX, 76-78). Il carattere di simulacro ossia non autentico, non puro delle apparizioni prefigurate in questa prima visione dell'Empireo viene ricondotto qui al topazio. Con ciò si rimanda all'effetto del riflesso, visto che nella gemmologia ai topazi è attribuita la capacità di essere buoni specchi ${ }^{21}$. Inoltre se ne mette in rilievo il potere curativo nel combattere malattie dell'occhio, usando il topazio per passarli sugli occhi in modo da rinforzarne la vista ${ }^{22}$. Beatrice afferma «[...] Non che da sé sian queste cose acerbe; / ma è difetto da la parte tua, / che non hai viste ancor tanto superbe». (Par. XXX, 79-81). E Dante reagisce: «[...] come fec' io, per far migliori spegli / ancor de li occhi, chinandomi a l'onda / che si deriva perché vi s'immegli» (Par. XXX, 85-87).

Il topazio, oltre che in questo brano, compare in tutta l'opera solo un'altra volta: come sineddoche ("vivo topazio») dell'anima di Cacciaguida, l'avo di Dante, nel Cielo di Marte (Par. XV, 85-87). In quel caso il topazio è incastonato in un prezioso gioiello, la radiosa Croce di gemme. Questa preziosità retoricamente e poeticamente prodotta simboleggia la stima dell'avo. Anche in questo caso Dante mostra un trattamento emancipato delle strategie retoriche e degli insegnamenti del suo maestro Brunetto Latini, il quale per la retorica consiglia di usare le parole come gemme preziose ${ }^{23}$. Dal punto di vista poetologico risulta evidente la simile struttura delle rime in cui la parola topazio è inserita in entrambi i brani nonché il parallelismo del concetto "sazio» $\mathrm{o}$ «sazi». Par. XV, 83-87: «ringrazio» (v. 83) - «topazio» (v. 85) - «sazio» (v. 87) e Par. XXX, 74-78: «sazi» (v. 74) - «topazi» (v. 76) - «prefazi» (v. 78).

Beatrice smaschera il panorama del fiume di luce, prima visione dantesca dell'Empireo, come illusione, come immagine «non matura» («queste cose acerbe», v. 79). Il visitatore anelante al supremo potrà ammirare tutta la vera maestà solo dopo che avrà reso i propri occhi degli specchi ancora migliori. A tal fine il bramoso pellegrino, nel suo ardente desiderio, inumidisce le ciglia degli occhi con l'onda del fiume di luce («chinandomi a l'onda / che si deriva perché vi s’immegli», v. 86-87). Da ciò scaturisce l'associazione delle parole di Beatrice «Il fiume e li topazi» (v. 76) e con essa una allusione al potere curativo che il topazio ha per gli occhi. Contemporaneamente, dal momento che tale gemma simboleggia anche l'amore per Dio e l'amore per il prossimo ${ }^{24}$, l'atto

21. Cfr. Albertus Magnus, De mineralibus, II, XVIII, voce «Topasion».

22. Cfr. U. Korzeniewski, Karfunkelstein und Rosenquarz. Mythos und Symbolik edler Steine, Ostfildern, Jan Thorbecke Verlag, 2005, p. 130, voce «Topas».

23. Cfr. Latini, Tresor, II, 1.2.

24. Cfr. Marbod de Rennes, Lapidum pretiosorum mystica seu moralis applicatio, IX, voce «Topasius»: «Significat eos qui Deum et proximum diligunt. Qui autem proximum diligunt, sicut aurum fulgent, et a sordibus huius saeculi purgati, et semper celestibus intendentes, clariores et pulchriores efficiuntur». (Cit. secondo l'edizione approntata da Bruno Basile: MARBODO DI RENNes, Lapidari. La magia delle pietre preziose, Roma: Carocci, 2006, p. 130). Sul rapporto di questo passo con i canti danteschi Par. XV, 85 e Par. XXX, 76 cfr. ivi, nota 13, p. 141. 
simbolico del bere per rinforzare la vista acquista la connotazione dell'intensificazione dell'amore per Dio da parte del pellegrino che lo cerca. È dato supporre che, dietro il riferimento all'onda "che si deriva» si celi un rimando all'origine divina del fiume ${ }^{25}$. La consistenza dell' "onda / che si deriva»e scorre non viene qui direttamente menzionata, sebbene vi riecheggi la precedente sollecitazione di Beatrice «ma di quest'acqua convien che tu bei» (v. 73) e nonostante il paragone con la sete di latte materno del lattante (v. 82-84) rimandi alla materna Maria ed alla forza vitale del latte. Il mistero di quest'atto di grazia dell'illuminazione, rispecchiato dai versi 85-89, appare a sua volta nell'ambivalenza tra acqua, latte e fiamme (fuoco) (cfr. supra). Esso allude sia al «fiume d'acqua viva» (Apocalisse 22, 1) sia alla Vergine Maria nonché al dono dello Spirito Santo. Come nei versi 68-72, che esprimono il desiderio del supremo, anche in questo caso, in relazione al metaforismo del desiderio nel momento del suo esaudimento, si mostra una densità espressiva nell'uso di pregnanti neologismi derivanti dal linguaggio dantesco («si deriva», «s'immegli») ${ }^{26}$.

Direttamente dopo questo mistero, sin dal cuore della terzina Par. XXX, 88-90, a partire dal secondo emistichio del secondo verso, ha inizio la seconda visione dantesca dell'Empireo: "così mi parve / di sua lunghezza divenuta tonda» (v. 89-90). Con la metamorfosi delle dimensioni del fiume di luce dalla linearità al cerchio, percepita da Dante, egli perviene alla capacità della visione mistica della Rosa. Dante riconosce il mascheramento della prima visione, che era solo una metafora.

\section{Il disegno di Botticelli per il Canto XXX del Paradiso: Approcci per un sistema di corrispondenze tra testo e immagine}

\subsection{La selezione del soggetto e il problema della non rappresentabilità}

All'incirca due secoli dopo Dante, un pittore del primo Rinascimento, Sandro Botticelli (1444/45 - 1510), si cimenta con l'impresa della trasposizione del testo del poema dantesco in un ciclo di disegni ${ }^{27}$. Nel caso del disegno botticelliano a illustrazione del Canto XXX del Paradiso (Fig. 1) si tratta dell'ultimo

25. Cfr. Chiavacci Leonardi, op. cit., commento ai versi 86-87, p. 545.

26. Cfr. Ibidem.

27. In merito ad una datazione precisa dei fogli, in gran parte custoditi presso il Kupferstichkabinett dei Musei statali di Berlino, si registrano ipotesi diverse. Cfr. l'introduzione di Hein-Th. Schulze Altcappenberg, 'per essere persona sofistica'. Botticellis Bilderzyklus zur Göttlichen Komödie, in ID. (a cura di), Sandro Botticelli. Der Bilderzyklus zu Dantes Göttlicher Komödie. Mit einer repräsentativen Auswahl von Zeichnungen Botticellis und illuminierten Commedia-Handschriften der Renaissance, Ostfildern-Ruit, Hatje Cantz e London: Royal Academy of Arts, 2000, pp. 14-35: 23-28. La maggior parte degli storici dell'arte fa risalire i disegni botticelliani del Paradiso agli anni Novanta del Quattrocento. 


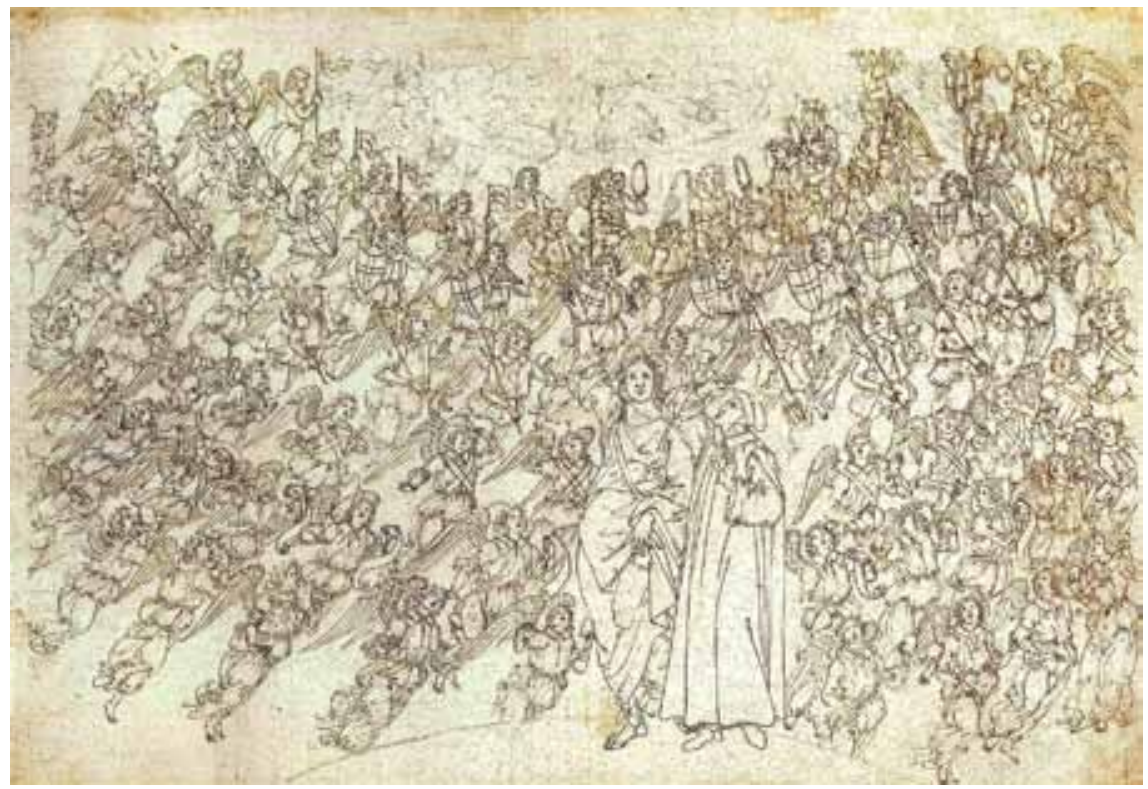

Figura 5. Sandro Botticelli (1444/45-1510), Paradiso XXVIII. Disegno facente parte del ciclo di illustrazioni della Divina Commedia di Dante. (Botticelli/Codex Hamilton 201).

() Kupferstichkabinett. Staatliche Museen zu Berlin.

foglio quasi compiuto del ciclo $^{28}$. Esso mostra la prima visione dell'Empireo, il fiume di luce come metafora e prefigurazione della Rosa dei Beati (cfr. Par. XXX, 61-78). Dall'intero Canto l'artista ha estrapolato questo piccolo brano di 18 versi che rappresenta un'immagine mentale come word-painting. Il mistero dell'illuminazione, che precede questa visione e viene descritto come pura esperienza di luce e trasformazione della capacità visiva (Par. XXX, 46-60), in altre parole: la prima parte del Canto, non trova trasposizione figurativa in Botticelli. Né viene rappresentata la seconda visione dell'Empireo, che dopo la trasformazione del fiume in un cerchio consente di guardare a occhi nudi la

28. I fogli per Par. XXXI e Par. XXXIII rimasero in bianco. Il foglio originariamente previsto per illustrare il Canto XXXI fu usato per la parte inferiore del Grande Satana (Inf. XXXIV, 2). Sotto le gambe di Satana si riconosce il palinsesto di una figura con diversi gradini che potrebbe essere lo schizzo di una Rosa celeste formata da tanti petali. Cfr. Schulze Altcappenberg, Der Bilderzyklus von Sandro Botticelli. Inferno, Purgatorio. Paradiso, op. cit., pp. 37-290: 284 (spiegazione di Par. XXXI). Per il foglio destinato a illustrare il Canto XXXIII Schulze Altcappenberg [cit., p. 288] prende in considerazione la possibilità che esista una "lacuna» voluta che potrebbe essere riconducibile al «riconoscimento dei limiti dell'immagine». Il foglio per il Canto XXXII mostra in miniatura così come a grande distanza, ma visto in piena messa a fuoco, un gruppo di tre figure identificabili con Maria (sinistra), Cristo con le stimmate (centro) e l'Arcangelo Gabriele (destra). Per ulteriori dettagli sull'interpretazione di questo disegno cfr. Schulze Altcappenberg, op. cit., p. 286. 
Rosa dei Beati, simulando con ciò il passaggio dalla temporalità all'eternità, all'infinità. Sembra impossibile per l'artista immaginare figurativamente i paradossi rappresentati nella poesia dantesca dai paragoni come indice della sospensione delle leggi naturali ${ }^{29}$.

La poesia dantesca degli ultimi Canti del Paradiso (dal XXX al XXXIII) contiene una serie di paragoni, metafore e immagini riconducibili in senso fisico ad una sospensione delle leggi naturali: sospensione di spazio e tempo, vicinanza e lontananza, sopra e sotto, ossia verticalità e orizzontalità, linearità e circolarità, interno e esterno, materia e spirito, qualità e quantità nonché annullamento delle differenze tra acqua e fuoco.

\subsection{Il visivamente immaginabile e la sovrannaturale metaforicità in Par. XXX, 61-96}

In questa sede analizziamo il word-painting del testo (Par. XXX, 61-78) dal punto di vista della sua sostanza visiva a disposizione del disegnatore. Dante introduce questa sequenza con un «vidi» (v. 61) e sottolinea questa impressione ottica con il participio «dipinte» (v. 63). Con ogni probabilità tale segnale testuale rappresentò uno spunto del tutto particolare per Botticelli. Le seguenti parole o gruppi di parole contengono impressioni visive o visualizzabili:

— «forma di rivera» (v. 61)

— «intra due rive» (v. 62)

— «mirabil primavera» (v. 63)

— «Di tal fiumana uscian faville vive» (v. 64)

— «e d'ogne parte si mettien ne' fiori» (v. 65)

- «quasi rubin che oro circunscrive» (v. 66)

— «riprofondavan sé nel miro gurge» (v. 68)

— «e s'una intrava, un'altra n'uscia fori» (v. 69)

— «Il fiume e li topazi» (v. 76)

— «ch'entrano ed escono e 'l rider de l'erbe» (v. 77)

Già questo catalogo dell'immaginabile visivo è infarcito di metaforicità sovrannaturale:

- Su v. 61/62: Le due rive delimitano un fiume di luce di splendore rossodorato.

- Su v. 63: I fiori primaverili («mirabil primavera») sono connotati con il simbolismo dell'eterna primavera, che per il pittore risulta difficilmente realizzabile.

- Su v. 64-66: Questa terzina offre un'immagine chiusa. La metamorfosi delle scintille in rubini e dei fiori in cornici d'oro traspone nella simulazione,

29. Andreas Kablitz nega la competenza dell'immaginazione alla conoscenza dantesca dell'Aldilà. Cfr. Kablitz zu Par. XXX, da verso 97 fino a verso 123, op. cit., pp. 245-246. 


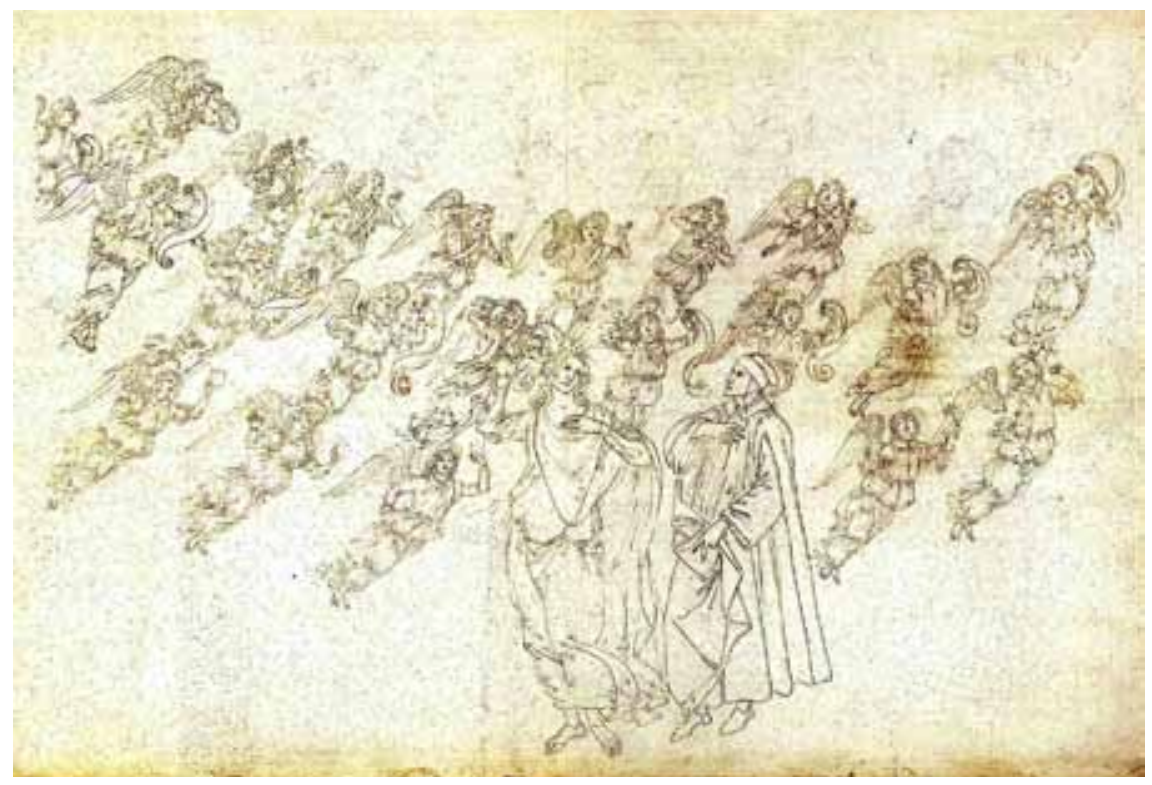

Figura 6. Sandro Botticelli (1444/45-1510), Paradiso XXIX. Disegno facente parte del ciclo di illustrazioni della Divina Commedia di Dante. (Botticelli/Codex Hamilton 201).

${ }^{\odot}$ Kupferstichkabinett. Staatliche Museen zu Berlin.

attraverso l'ibridizzazione «faville vive» = «rubin» $\mathrm{e}$ «fiori» = «oro», un cambio di valore dal vivo/effimero all'eterno.

- Su v. 68/69: Se si intende «miro gurge» come mirabile gorgo di luce all'interno del fiume di luce, si dà in certo modo un legame 'logico' con il reimmergersi delle «faville vive»; d'altro canto solo a patto che i movimenti rappresentati fin lì e le impressioni visivamente percepibili vengano considerati come un gioco di luce e raggi: fuoco, gemme riflettenti, oro splendente e gorgo mirabile nel senso di luce.

- Su v. 70-75: Questi versi contengono un discorso di Beatrice codificato sotto forma di metafore con la sollecitazione per Dante di spegnere la sua sete, ossia il suo desiderio sovrannaturale, con questo fiume. Con «ma di quest'acqua convien che tu bei» (v. 73) Beatrice si serve di una immagine terrena per un atto simbolico rivolto al metafisico. Il verso conclusivo fa ricorso alla finora significativa interazione degli sguardi tra Dante e Beatrice («il sol de li occhi miei», v. 75). Lo scambio di sguardi tra i due protagonisti rappresenta un importante motivo artistico in tutti i fogli di Botticelli dedicati al Paradiso. In molti disegni esso (ossia lo scambio di sguardi) appare l'unico mezzo per realizzare il movimento mentale del testo.

- Su v. 76-78: Questa terzina che costituisce il finale della prima visione rappresenta a sua volta una sfida per l'artista nella misura in cui Beatrice presenta le cose precedentemente descritte - il fiume, le faville e i fiori - 
solo come un'adombrante prefigurazione della verità. Con ciò essa dichiara quanto visto essere un'illusione, un simulacro: «son di lor vero umbriferi prefazi» (v. 78). Per giunta la rima «topazi» (v. 76) - «prefazi» (v. 78) suggerisce che si tratta solo di un riflesso della Verità perché il topazio è solo uno specchio ${ }^{30}$.

Il verso 67 («inebrïate da li odori»), che rappresenta un'impressione olfattiva, sembrerebbe inizialmente inadeguato ad essere rappresentato con il linguaggio delle immagini del disegnatore.

Ci si chiede, dunque, come faccia l'ingegno dell'artista figurativo, con i suoi strumenti ed il suo linguaggio delle immagini, a trasporre il word-painting scaturito dall' «alta fantasia» (Par. XXXIII, 142) del poeta. Come può egli dare forma alla sfera immateriale dell' Utopos e rappresentare i movimenti dell'anima di chi è in cerca della Verità ${ }^{31}$ ?

\subsection{La concezione spaziale e la composizione del contenuto dell'immagine}

Sul foglio del disegno per il Canto XXX del Paradiso Botticelli continua in certo qual modo il suo schema di costruzione progettato per la terza Cantica. Il foglio per il Canto II (Fig. 2) mostra il modello topografico del Cosmo sotto forma di semplice disegno a compasso raffigurante cerchi concentrici tutti intorno alla terra. Da questo modello scaturisce per così dire la struttura spaziale alla base della Cantica. Fino al disegno per il Canto XX, servendosi di una

30. Sui topazi come specchi cfr. supra, paragrafo 2.2.3.

31. Un confronto dettagliato testo-immagine tra il Canto XXX del Paradiso e il corrispondente disegno di Botticelli secondo l'approccio specifico del nostro studio non risulta essere stato finora avanzato. Cfr. le rappresentazioni complessive di Schulze Altcappenberg, 'per essere persona sofistica', op. cit., pp. 23-28 (spiegazione del foglio di Par. XXX, op. cit., p. 282); Damian Dombrowski, Botticelli und die Konstrukion des Geistes. Die Dante-Zeichnungen und die Grenzen der Kulturgeschichte, in Schulze Altcappenberg, op. cit., pp. 298-305; Botticelli e Dante, a cura di Corrado GizzI, Milano: Electa, 1990 (contiene una breve annotazione sul foglio di Par. XXX, op. cit., p. 317); Kenneth Clark, The Drawings by Sandro Botticelli for Dante's Divine Comedy after the Originals in the Berlin Museums and the Vatican, London: Thames and Hudson, 1976; Bernard Berenson, I disegni dei pittori fiorentini, 3 voll., Milano: Electa, 1961 (un giudizio avvilente del ciclo botticelliano dato in base ad un punto di vista superficiale [cfr. p. 146]; nessuna trattazione specifica di Par. XXX); Yvonne Batard, Les dessins de Sandro Botticelli pour la Divine Comédie, Paris: Perrin, 1952 ("Collection Jeu savant», 2), p. 107 (una semplice annotazione sul foglio XXX); Igino B. Supino, I disegni per la Divina Commedia di Dante, Bologna, 1921; Friedrich Lippmann (a cura di), Zeichnungen von Sandro Botticelli zu Dantes Göttlicher Komödie. Verkleinerte Nachbildungen der Originale im Kupferstichkabinett zu Berlin und in der Bibliothek des Vatikans, mit einer Einleitung und der Erklärung der Darstellungen, Berlin: Grote, $1921^{2}\left(1887^{1}\right)$, p. 73; Adolfo Venturi, Il Botticelli interprete di Dante, Firenze: Le Monnier, 1921, pp. 133-134 (una descrizione sommaria ed entusiastica della scena dell'immagine botticelliana senza approccio interpretativo e senza alcun riferimento al poema dantesco). Cfr. anche Herma Bashir-Hecht, "Die Paradieslandschaft bei Dante und Botticelli», Die Kunst, 97 (1985), pp. 1031-1034: 1034 (improduttivo per un'analisi scientifica). 
semplice linea circolare chiusa, Botticelli allude di volta in volta al rispettivo cielo planetario. Con l'ascesa al Cielo delle stelle fisse sulla scala di Giacobbe, sui fogli XXI e XXII (Fig. 3-4), la scala celeste viene obliquamente iscritta nella circonferenza del cerchio e diretta verso l'alto. L'estremità superiore della scala sul foglio XXII oltrepassa il limite della circonferenza del cerchio. Sui disegni a partire da Par. XXIV fino al Canto XXIX (Primo Mobile) incluso la circonferenza appare ristretta ad un semicerchio sul margine inferiore del foglio ${ }^{32}$, la cui superficie continua ad offrire la piattaforma per le figure di Beatrice e Dante. Questi disegni rappresentano i Cori angelici prima come esseri avvolti di luce sotto forma di faville («cerchio d'igne», Par. XXVIII, 25), poi, sui fogli XXVIII e XXIX (Fig. 5-6), come figure corporee, differenziandosi così dal testo dantesco ${ }^{33}$.

Solo con il passaggio dal Primo Mobile all'Empireo, ossia dal mondo materiale alla sfera immateriale del cosiddetto Cielo di fuoco, il pittore fa sparire la linea circolare, rinunciando nel Canto XXX (Fig. 1) a dare l'illusione di una solida base sotto i piedi. Ciò si può interpretare come il tentativo di suggerire il venir meno della spazialità a favore dell'infinità. Pur tuttavia il disegno riflette un'archetipica - soprattutto cristiana - idea spaziale di movimento verso l'alto ben radicata nell'immaginativa umana. Botticelli si ricollega alla sua costruzione della Scala santa (Par. XXI e XXII) (Fig. 3-4), tracciando, a una certa distanza l'una dall'altra, due linee diagonali da in basso a destra a in alto a sinistra, destinate ad incontrarsi al di là della superficie del disegno (vaga allusione ad una sorta di prospettiva). Con queste linee egli allude ai contorni del fiume di luce attraverso le 'rive' ("forma di rivera», Par. XXX, 61). Le linee di contorno schizzate fanno intuire il centro in un punto imprecisato che cade al di fuori del foglio. Su questo foglio le abitudini visive perspettivistiche appaiono disturbate. Per l'artista rinascimentale ciò dovrebbe reputarsi come sinonimo della rinuncia a una rappresentazione spaziale che dia l'illusione della realtà. Con ciò egli rende conto della visione trascendente del testo poetico, per la quale, a partire da Par. XXX, non valgono più le leggi della natura ${ }^{34}$.

\section{Il panorama: fiume di luce e mirabil primavera}

\subsection{La 'riva' e le "faville vive»: Permutazione e 'quadro con segreto'}

Le figure di Dante e Beatrice, nella metà inferiore destra dell'immagine, non insistono su alcuna base. Poiché i loro piedi non poggiano su nessuna superficie, esse sembrano librarsi in volo al di sopra del fiume di luce. Sulla 'riva' sinistra troviamo una superficie riccamente decorata con elementi vegetali composti da circa tredici rigogliosi vegetali di fantasia a forma di fiori di diver-

32. Sul foglio che illustra Par. XXVII il semicerchio è solo intuibile.

33. Sebbene Dante in Par. XXVIII, 25 usi l'espressione «cerchio d'igne», su questo e sul foglio successivo Botticelli disegna delle figure e non delle fiamme.

34. Cfr. anche Kablitz, op. cit., pp. 242 ss. 
sa foggia («mirabil primavera», Par. XXX, 63). Essi sono sparsi di qua e di là e non costituiscono alcun punto d'orientamento spaziale di un sopra o un sotto (Fig. 7: dettaglio, v. Fig. 1). Forme simili si possono riconoscere come disegno a stilo nella metà superiore destra dell'immagine al di sopra della diagonale superiore («riva»), quasi come panorama floreale sulla 'riva' opposta ${ }^{35}$. Tra le due linee che delimitano il fiume («intra due rive», Par. XXX, 62), a sinistra sopra le figure di Dante e Beatrice, scopriamo dodici minuscoli creature sporadicamente distribuite, che di primo acchito sembrano ermetiche ma che a meglio guardare si riesce a identificare. Si tratta della trasposizione figurativa delle «faville vive». Botticelli ha 'vivificato' nel vero senso della parola le faville dell'espressione poetica, rappresentando piccoli angioletti ${ }^{36}$ alati avvolti in faville o raggiere (Fig. 8: dettaglio, v. Fig. 1). In queste minicomposizioni le figure sortiscono l'effetto di piccoli 'quadri con segreto' alla Arcimboldo, vexierbilder, apparendo nelle faville a volte orizzontali, altre volte oblique, capovolte, ritratte di faccia, di spalle o di profilo. Oltre a questo gruppo ci sono anche figure non avvolte in faville o raggiera. Esse sono in parte disegnate a figura intera, in parte solo a mezzo busto, in altri casi solo dalla vita in giù, a gambe tese verso l'alto. Questa varietà delle dodici piccole figure al di sopra del fiume di luce virtuale allude alla dinamica del librarsi, dell'immergersi, del riemergere e del continuare a librarsi: «Di tal fiumana uscian faville vive» (Par. $\mathrm{XXX}, 64$ ); «riprofondavan sé nel miro gurge» (v. 68); «e s'una intrava, un'altra n'uscia fori» (v. 69).

Osservando l'originale, conservato presso il Kupferstichkabinett di Berlino, con l'aiuto di una lente d'ingrandimento e confrontandolo con la riproduzione, si ha una sorpresa: l'intero fiume di luce è popolato da figure angeliche che specialmente nella parte inferiore del fiume restarono non completamente elaborate. Se ne trovano addirittura alcune tra Dante e Beatrice che svolazzano intorno alle loro teste o fanno capolino da sotto i piedi di Dante. Nella parte superiore del fiume di luce il margine superiore è ricoperto di angeli che si gettano a capofitto nella corrente. Questo modo di comporre l'immagine richiama l'idea della vivacità e dell'abbondanza.

35. L'artista si è qui limitato ad un abbozzo preparatorio con lo stilo. Il disegno a mano copre l'intero foglio, eccezion fatta per un piccolo triangolo formato dal limite del fiume di luce e il margine superiore del foglio che è rimasto bianco. Così, il disegno a inchiostro per Par. XXX non risulta completamente eseguito. Sulla tecnica di lavoro botticelliana e sulla differente elaborazione dei fogli del ciclo cfr. Doris Oltrogge, Robert Fuchs, Oliver Hahn, «Finito und Nonfinito. Zur Technik von Zeichnung und Malerei in Botticellis Divina Commedia», in Schulze Altcappenberg (a cura di), Sandro Botticelli, op. cit., pp. 334-341.

36. Si tratta di un riferimento intramediale alle figure che volano intorno alla scala celeste sul foglio per Par. XXI. Cfr. Schulze Altcappenberg, Der Bilderzyklus von Sandro Botticelli. Inferno. Purgatorio. Paradiso, in ID. (a cura di), Sandro Botticelli, op. cit., p. 258. Per motivi di corrispondenza con il testo dantesco, a proposito dell'illustrazione botticelliana preferiamo in questa sede utilizzare il termine "angeli» piuttosto che «geni». 


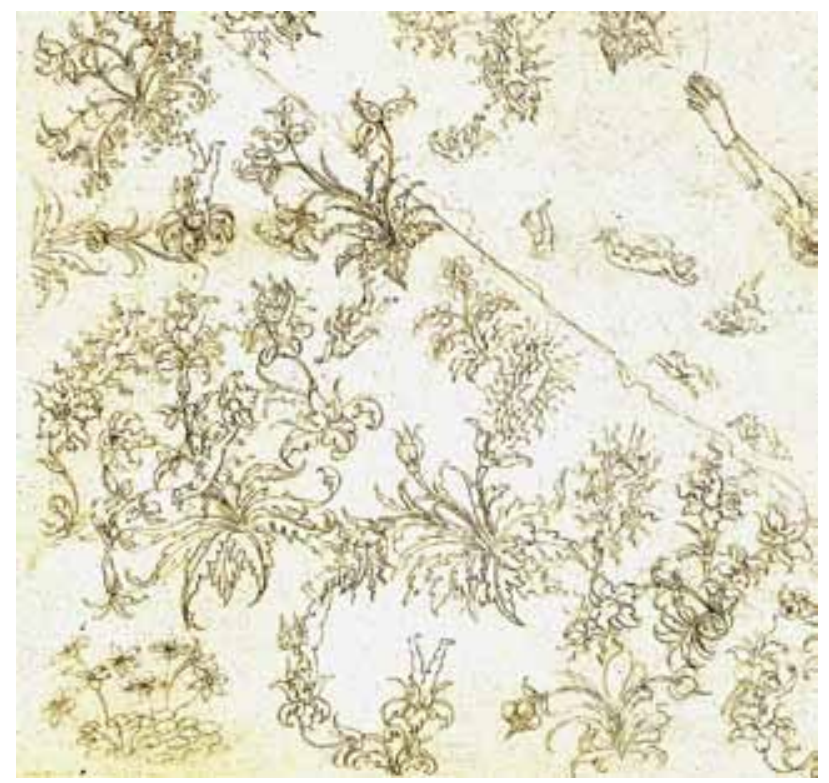

Figura 7. Sandro Botticelli (1444/45-1510), Paradiso XXX. Disegno facente parte del ciclo di illustrazioni della Divina Commedia di Dante. (Botticelli/Codex Hamilton 201). Ingrandimento di un particolare del panorama floreale in basso a sinistra. ${ }^{\odot}$ Kupferstichkabinett. Staatliche Museen zu Berlin.

\subsection{Strategie del disegnatore Botticelli: differenze e corrispondenze rispetto al Poema}

Il disegno del panorama floreale in 'riva al fiume' sembra riuscito del tutto all'altezza del modello poetico («mirabil primavera», v. 63). Ciò appare evidente ad un più attento esame della composizione del suddetto 'quadro con segreto' o vexierbild. Foglie, fiori, gambi sono fusi con minuscole figure angeliche sorprendentemente riconoscibili, con poche eccezioni, in ogni pianta. L'intera superficie dell'immagine nella parte inferiore sinistra e superiore destra appare come ricoperta da una fitta decorazione arabescata. Similmente alle creature descritte sul fiume di luce, gli angeli sono talvolta avvolti da fiamme (in volo verso un fiore), ma spesso anche nudi. Anche per queste figure vale quanto abbiamo già osservato a proposito della varietà della direzione e dei movimenti ("e d'ogne parte si mettien ne' fiori», v. 65). Alcuni corpicini spuntano solo con le gambe dal calice di un fiore in cui si sono immersi a capofitto ${ }^{37}$.

37. Nel sistema delle corrispondenze del ciclo di disegni botticelliano gli angeli immersi nei fiori di Par. XXX rievocano una reminiscenza intramediale dell'illustrazione di Inf. XIX raffigurante la pena dei simoniaci inseriti a testa in giù in buche nel terreno da cui fuoriescono solo le gambe, che essi agitano mentre fiammelle vive gli guizzano sulle piante 
Mentre singole figure del disegno botticelliano sono rappresentate a mezzo busto nell'atto di ergersi dai fiori, altre figure sembrano compiere esercizi acrobatici - come per esempio una verticale sulle mani o sulla testa, stando ritte su un fiore - oppure penzilano aggrappate allo stelo. Le figure danno l'impressione di quello che possiamo definire un «sentirsi-attratti-dai-fiori». La varietà e la quantità dei loro movimenti sottolinea l'effetto della leggerezza e del ludico, di una accentuata vogliosità e di una indomabile energia vitale. Da quanto osservato si deduce che in questa scena artistica si può vedere una prefigurazione dei versi 4-21 di Par. XXXI, ossia la metamorfosi degli angeli in uno sciame di api. Sotto quest' aspetto del dolce anche Par. XXX, 67 («inebrïate da li odori») acquista significato per il pittore. Infatti, considerando le teste, o addirittura i corpi degli angeli immersi nei calici dei fiori, si è spinti a fare un'associazione con l'ebbrezza e con una indomabile delizia, nonché con il bisogno di inebriarsi del profumo dei fiori.

Il panorama dei fiori e degli angeli sulla 'riva' del fiume di luce richiama, nei suoi elementi vegetali, gli ornamenti che arricchiscono l'abito di Flora nel dipinto botticelliano de La Primavera. Il panorama fantastico di foglie, fiori, calici e ovari nonché le parti del corpo degli angeli che vi fuoriescono, di cui alcuni per così dire 'sbocciano' nei fiori, offrono all'occhio dell'osservatore l'illusione di stare ammirando forme floreali tempestate di gemme preziose, creazioni simili a gioielli ("quasi rubin che oro circunscrive», v. 66). L'impressione dello scintillìo e dello splendore viene accentuata attraverso le faville che circondano le figure, col che si può in certa misura giustificare anche un rapporto con l'illustrazione dei versi 76 e 77 («Il fiume e li topazi» e «ch'entrano ed escono e 'l rider de l'erbe»).

\subsection{Rinuncia alla trasposizione dell'allegoresi delle gemme preziose: forma invece che colore}

L'analisi consente di constatare che nella sua rappresentazione del fiume di luce e della «mirabil primavera» Botticelli ha trasposto figurativamente le impressioni $^{38}$ visive e visualizzabili evocate dal testo dantesco ai versi 61-77. Non chiarita resta tuttavia la questione dei colori rosso rubino, oro e topazio. La letteratura storico-critica ovvero critico-artistica rivela posizioni controverse in merito alla domanda se per il ciclo botticelliano fosse o meno prevista una colorazione. Lo stato attuale della ricerca è ipotetico e non ci permette di

dei piedi. In tale contesto trovano menzione tre importanti papi: Nicola III, che sconta la sua pena nella buca, Bonifacio VIII, che presto lo seguirà, e il suo successore Clemente V. Non va taciuto che gli ultimi versi del XXX del Paradiso stigmatizzano ancora una volta la simonia e contengono un'allusione a Bonifacio VIII, nel frattempo deceduto, e a Clemente V, che presto lo seguirà all'Inferno. Ciò è un indizio del fatto che il disegnatore Botticelli si è voluto accostare molto al testo poetico, più di quanto non possa sembrare a prima vista.

38. Cfr. la lista supra, nel paragrafo 3.2. 
argomentare da un sicuro punto di partenza ${ }^{39}$. In questa sede possiamo solo fare riferimento all'allestimento e alla forza espressiva del disegno nello stato in cui lo abbiamo, senza, quindi, considerare un'eventuale colorazione. Le superfici in corrispondenza a destra e a sinistra della 'riva' sono marcatamente ricalcate con un intreccio di sottili tratti e linee (trattini d'inchiostro o stilo) che rappresentano oggetti. Tanto come disegno a inchiostro nella parte inferiore quanto come disegno a stilo nella parte superiore essi non sortiscono affatto l'effetto di uno schizzo. La forza espressiva dell'intreccio delle forme minuziosamente elaborate con linee e trattini si può solo difficilmente superare con accenti cromatici. Ciò vale anche per l'esecuzione del disegno delle figure di Dante e Beatrice ${ }^{40}$.

In questo caso il potere simbolico della gemma rossa, a cui nel disegno botticelliano non corrispondono effetti cromatici, si esprime solo indirettamente attraverso il linguaggio delle forme. Né il foglio a illustrazione del XXX del Paradiso né tanto meno gli altri disegni di Botticelli a illustrazione della Cantica offrono punti di appoggio per una diretta corrispondenza nei confronti dell'allegoresi delle pietre preziose, pur così costitutiva della mistica dantesca del Paradiso. Questa differenza intermediale è spiegabile probabilmente con i ricorrenti rimandi di Dante agli effetti della "pura luce». Le esperienze luminose 'elementari', evocate dal Poeta come illuminazione interiore, non sono alla portata delle possibilità figurative dell'artista rinascimentale ${ }^{41}$. La straordinaria intensità della luminosità del rubino viene dirottata dal disegnatore nella spumeggiante vivacità e dinamica delle figure angeliche. L'emanazione di

39. Sull'ipotesi secondo la quale i disegni botticelliani fossero destinati ad essere successivamente miniati cfr. Aldo Bertini, I grandi maestri del disegno. Botticelli, Milano, 1953, p. 11 e sulla sua scia Paolo Bellini, Le due serie di disegni del Botticelli per la Commedia, in Botticelli e Dante, cit., pp. 41-50: 47. Cfr. anche Oltrogge, Fuchs, Hahn, Finito und Nonfinito. Zur Technik von Zeichnung und Malerei in Botticellis Divina Commedia, in Schulze Altcappenberg, op. cit., p. 338 e p. 341 . Contro l'ipotesi di disegni da miniare si schiera, invece, LAMBerto Donati (Il Botticelli e le prime illustrazioni della Divina Commedia, Firenze: Olschki, 1962, p. 15).

40. Cfr. infra, paragrafi 5.1. e 5.2.

41. Un miniaturista della prima metà del Trecento, come quello del Codex Egerton 943 (British Library, London) realizzato a Bologna, non aveva evidentemente alcun esitazione a realizzare l'immagine dantesca del fiume luminoso e della «mirabil primavera» (p. 179 v.; cfr. Fig. 9). Il pittore prende chiaramente a motivo il verso 76 («Il fiume e li topazi»), componendo una figura in cui sullo sfondo misticamente blu del fiume luminoso spicca un certo numero di forme che somigliano a bottoni («li topazi») color oro incastonati in una cornice rossa che irradiano raggi: un'allusione al verso 66 ("quasi rubin che oro circunscrive»). L'inversione di rosso (qui esterno) e oro (qui interno), tuttavia, è indice del fatto che, nella realizzazione della mistica della luce delle pietre preziose, l'artista tocca il suo limite. Così, egli trasforma le gemme del verso dantesco in ambigui segni ermetici che ricordano anche delle stelle, così che il fiume di luce blu tra le 'rive' suggerisce l'immagine di un celeste paesaggio paradisiaco. La cornice rossa dei 'topazi d'oro', inoltre, rinvia allo splendore rosso oro del fiume luminoso (verso 62: «fulvido di fulgore»). Le due 'rive' di quest'ultimo prefigurano l'apertura della Rosa. La miniatura sortisce il suo effetto grazie ai colori, mentre la maggiore forza espressiva insita nel tratteggio dei disegni botticelliani del Paradiso non sembra necessitare di una aggiuntiva elaborazione cromatica. 


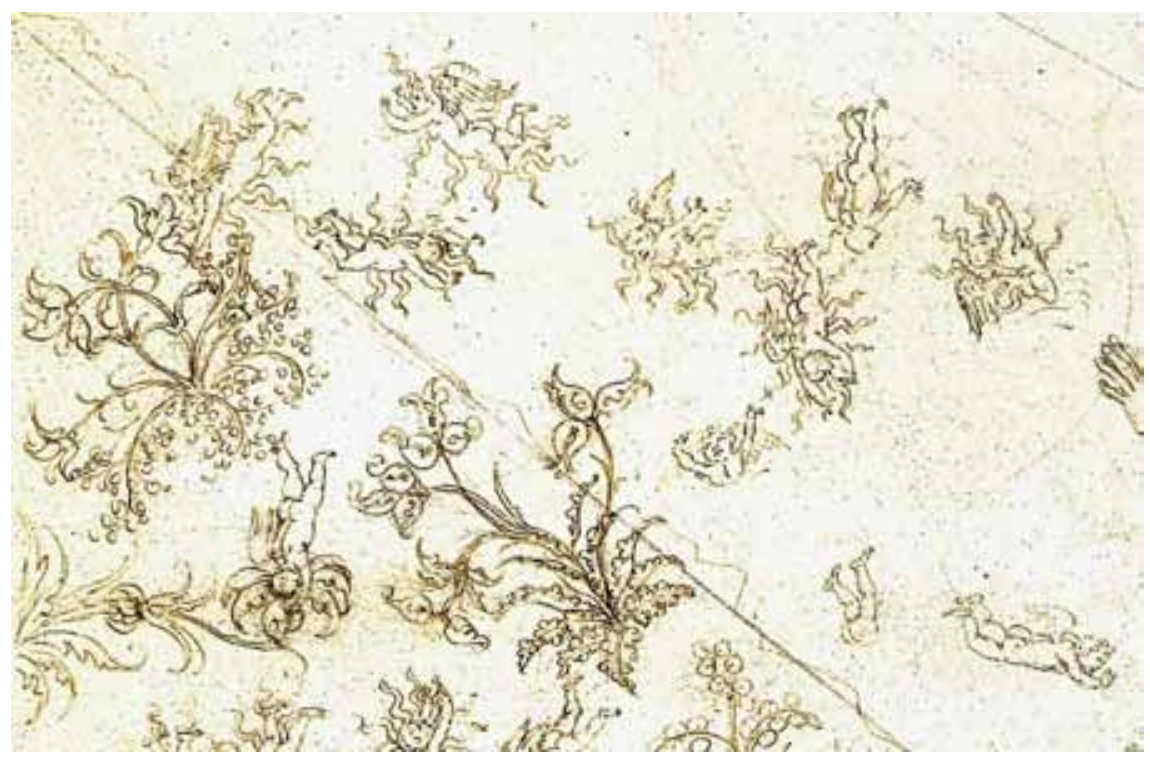

Figura 8. Sandro Botticelli (1444/45-1510), Paradiso XXX. Disegno facente parte del ciclo di illustrazioni della Divina Commedia di Dante. (Botticelli/Codex Hamilton 201).

Ingrandimento di un particolare del fiume di fuoco con angeli circondati da raggiera.

${ }^{\circ}$ Kupferstichkabinett. Staatliche Museen zu Berlin.

calore e l'ardente fuoco interiore dell'amore, simboleggiati dalla gemma, sono trasferiti nel linguaggio del corpo delle piccole figure e nelle faville e raggiere che le avvolgono. I corpi delle figure angeliche rappresentano il desiderio ardente di unirsi ai fiori (= i Beati) («quasi rubin che oro circunscrive», v. 66). Alla preziosità della metafora delle gemme retoricamente prodotta corrisponde in Botticelli il virtuosismo del raffinato tratteggio e la dinamica dei movimenti stilizzata in ornamento, cosa che a sua volta rappresenta una forma di preziosità.

\section{La rappresentazione dei protagonisti: la forza espressiva dei disegni}

\subsection{Beatrice: mezzi figurativi di una convincente trasposizione}

Beatrice e Dante sono sistemati nella metà inferiore destra dell'immagine. Il pittore li raffigura mentre si librano/volano al di sopra del fiume di luce. Anche qui, come negli altri fogli, si mostrano piccole differenziazioni nella rappresentazione della coppia rispetto agli altri disegni del Paradiso. La figura di Beatrice, che sembra dominare tutto il resto, si è spostata al centro dell'immagine. La sua testa costituisce per così dire il centro dell'intera composizione. Il braccio sinistro, sproporzionatamente lungo, indica in anticipo la direzione del movimento della coppia verso su a sinistra, il suo estendersi alla metà sinistra dell'immagine ne fa una istanza centrale per una dinamica in ascesa. Il braccio 
destro, coperto dall'abbondante drappeggio del vestito, consente agli sguardi dell'osservatore solo di osservare la parte inferiore e la mano leggermente piegata all'indietro. Il corpo di Beatrice è avvolto da una abbondante nuvola di stoffa le cui pieghe, in parte a sboffo, in parte svolazzanti, in parte drappeggianti, sembrano simulare un movimento in sospeso. Il disegno del vestito mostra nella delicatezza del tratteggio una affinità con il tappeto di fiori, anche se il tratteggio risulta meno dettagliato. Solo i lunghi riccioli si pongono in riferimento con le forme delle faville e della luce che avvolge gli angeli. La figura di Beatrice appare tutto sommato stranamente avvitata su se stessa. Il corpo vestito e le membra sono da intendersi come una vista di spalle, laddove solo i piedi che librano paralleli appaiono di profilo. Contrariamente ai piedi, la testa è rivolta all'indietro, così da mostrare solo il profilo del viso. Lo sguardo è indirizzato verso il volto di Dante.

A Botticelli bastano due semplici accorgimenti per esprimere qualcosa di decisivo per l'immagine di questa scena: Essa infatti rappresenta niente di meno che l'addio di Beatrice, la quale, sospesa, si sottrae al suo protetto. Osserviamo per prima cosa la testa girata all'indietro e leggermente rivolta in giù verso Dante; secondo: l'avambraccio destro con la mano ripiegata che accenna ad un saluto. Una parte dell'avambraccio e della mano viene leggermente tangere la manica dell'abito di Dante, la qual cosa è senz'altro da interpretare come segno che la figura di Dante non si trova accanto a Beatrice ma poco dietro di lei. Il sottrarsi di Beatrice, discretamente suggerito dal disegno botticelliano, corrisponde al testo del Canto XXX del Paradiso nella misura in cui in esso Beatrice compare per l'ultima volta come guida di Dante (Par. XXX, 124-148) e in Par. XXXI, 55-60 è di colpo sparita. Degno di menzione appare anche qui il fatto che l'occhio destro di Beatrice, che appare ingrossato e fortemente accentuato grazie a un tratto orizzontale al di sotto della cavità oculare, mostra nuovamente quella caratterizzazione innaturale osservabile su alcuni fogli e che rinvia al magico sguardo di smeraldo ${ }^{42}$. Anche se in questo caso non appare esservi una comunicazione di sguardi tra le due figure.

La composizione botticelliana per Par. XXX sposta la figura di Beatrice tra il mirabile paesaggio e Dante. Questa sistemazione, unitamente allo sguardo rivolto a Dante, rimanda al ruolo di mediatrice ricoperto da Beatrice. Allo stesso tempo, la speciale tecnica di disegno dell'artista suggerisce l'affinità o meglio l'appartenenza di Beatrice a questa prefigurazione, ossia a questa forma mascherata della Rosa dei Beati, verso cui ella sembra recarsi. Quest'impressione nasce attraverso l'ambivalenza tra la direzione del braccio sinistro indirizzato verso su e la direzione delle gambe (dall'altezza del ginocchio), senz'altro riconoscibile nonostante il vestito che le avvolge, e dei piedi che, superando la linea della «riva» sinistra, toccano già la zona della «mirabil primavera» alias Candida Rosa. Così, nei modi della sua 'narrazione' figurativa in fasi, l'artista allude al prossimo immergersi nella Rosa da parte di Beatrice e Dante : «Nel giallo de la rosa sempiterna, / che si digrada e dilata e redole / 
odor di lode al sol che sempre verna, / qual è colui che tace e dicer vole, / mi trasse Bëatrice» (Par. XXX, 124-128). Rispetto al Canto XXXI si mostra, inoltre, un'allusione al ritorno di Beatrice al suo posto in Paradiso.

\subsection{Dante: Segnali della raffigurazione dell'esperienza' sovrannaturale}

Anche l'immagine di Dante, nella parte inferiore destra del foglio, è rappresentata di spalle mentre, poco dietro a Beatrice, si libra in volo sul fiume di luce. Come la sua guida, anche il pellegrino che anela ad ascendere ha le braccia alzate. I movimenti del braccio sembrano però niente affatto orientati verso la meta finale come quelli dell'accompagnatrice. Entrambi gli avambracci sono flessi rispetto alla posizione orizzontale della parte superiore delle braccia; mentre le dita delle mani aperte sono leggermente divaricate. Ciò sottolinea, insieme ai piedi nudi, la posizione in sospeso della figura, ma d'altro canto allude anche a un gesto di preghiera in cui sembrano esprimersi il massimo disio e umiltà. Come già la testa di Beatrice, anche quella di Dante è raffigurata di profilo, il suo sguardo estasiato, rivolto verso l'interno, non è più diretto, come in molti altri disegni precedenti, verso gli occhi di Beatrice ma verso la visione della "mirabil primavera», ossia verso quel simulacro che gli sta per così dire come maschera davanti agli occhi prima che egli sperimenti il mistero dell'umidificazione degli stessi con il fiume di luce (Par. XXX, 85-96). Del resto il tratteggio eseguito dall'artista fa apparire il vestito del poeta floscio e, per così dire, privo di espressione, cosa che dà l'impressione dell'assenza del corpo sotto le vesti e di una immaterialità dell'evento in merito a quanto va compiendosi nel personaggio al di là della materialità.

Attraverso il linguaggio degli occhi e la posizione delle braccia corrono essenzialmente due linee di forza: riconosciamo anzitutto una diagonale obliqua alle due rette del fiume di luce che, partendo dagli occhi della figura dantesca corre obliquamente nella superficie inferiore sinistra, ossia nella «mirabil primavera» che riflette la visione del poeta ancorata all'aldiquà. Il secondo movimento consiste in un semicerchio formato dalle quattro braccia che attraverso il braccio sinistro teso di Beatrice simula il movimento della corrente luminosa e con ciò il movimento verso l'alto ossia verso le sfere superiori. La direzione dello sguardo di Beatrice, per così dire, costringe la figura di Dante ad andare avanti proseguendo verso le regioni celesti superiori. La composizione delle braccia e degli sguardi dà l'impressione di una accumulazione di energia. Il semicerchio accennato con la decisa tendenza verso l'alto simula un'energia motrice sovrannaturale che nell'ambivalenza tra anelare a procedere e farsi trasportare si oppone alla legge della gravità terrestre.

\section{Conclusione}

\section{Il disegno di Botticelli per Par. XXX: la realizzazione artistica di una metaforicità sovrannaturale}

Sulla base di un minuzioso paragone del Canto XXX del Paradiso con il disegno botticelliano che lo illustra possiamo formulare primi approcci per un sistema 
di corrispondenze sviluppato da Botticelli per la sua trasposizione figurativa del testo poetico. L'Empireo, la sfera smaterializzata, in qualità di Utopos si sottrae ad ogni classico schema di costruzione spaziale. Di conseguenza, il disegnatore rinuncia alla sistemazione spaziale come le linee circolari o semicircolari accennate nei precedenti fogli per rendere il Primo Mobile. Per le figure di Beatrice e Dante, rappresentate mentre si librano in volo, manca qualsivoglia riferimento ad un punto di appoggio spaziale. La struttura della prospettiva centrale è minata e presente solo come reminiscenza a favore di vaghi 'segnali di direzione' che suggeriscono un movimento verso l'alto.

Con le diagonali che vanno da in basso a destra a in alto a sinistra e l'immaginaria, opposta diagonale della direzione dello sguardo di Dante (da in alto a destra a in basso a sinistra) lo schema compositivo dell'immagine riflette le virulente opposte tendenze nel testo tra movimento verso l'alto del pellegrino in cerca di Dio e l' 'abbacinamento' dell'uomo (Dante) ancorato nell'aldiquà. Il semicerchio formato dalle quattro braccia, sbilanciato dal braccio di Beatrice steso in diagonale, corrisponde alla visione mistica dell'apertura del fiume di luce al tondo della Rosa dei Beati («così mi parve / di sua lunghezza divenuta tonda", Par. XXX, 89-90). Nello schema compositivo botticelliano il semicerchio che figura al centro dell'immagine, come impulso decisivo della composizione, suggerisce il mistero della vera visione della Rosa dei Beati.

L'analisi dei dettagli e degli arrangiamenti delle forme rappresentate rivela una sensibile compenetrazione di Botticelli nel mondo spiritual-religioso del Canto e nella sua poetica. Basandosi su dettagliate indicazioni contenute nel testo, l'artista traspone figurativamente con grande esattezza l'intenzione di un intero brano del Canto XXX (la prima visione: fiume di luce e «mirabil primavera», v. 61-78). Ciò facendo, egli sposta la metaforicità sovrannaturale dei versi danteschi attraverso una deviazione. Tale differenza viene realizzata attraverso la concretizzazione dell'espressione simbolica "faville vive» che Botticelli trasforma in angeli, in angeli avvolti in faville e nelle raggiere. Da questa concretizzazione scaturiscono per l'artista ulteriori possibilità di riferimenti intermediali e intramediali, per esempio gli angeli avvolti nelle faville e nelle raggiere appaiono come delle prefigurazioni dello sciame d'api del Canto XXXI oppure come creature simili a gioielli sulle forme floreali, tempestate di gemme, delle piante fantastiche. Questa concretizzazione sotto forma di angeli le cui gambe fuoriescono dai calici dei fiori conduce anche ad una analogia di immagine con i Papi simoniaci evocati alla fine del Canto XXX del Paradiso che nel XIX dell'Inferno appaiono costretti a stare a testa in giù nella stessa buca.

La ricca e riccamente decorata superficie della «mirabil primavera» si rivela in Botticelli come un prisma di molteplici segnali. Il panorama di fiori, angeli e faville riflette abbondanza, diversità, vivacità, assenza di direzione, mancanza di orientamento spaziale, un caos 'innocente' e spensierato. L'ermetica del testo dantesco appare in questo caso trasposta nella ambivalenza tipica del 'quadro con segreto' o vexierbild consistente qui in un labirinto di forme che sortisce un effetto di mistero. In questo contesto si può riconoscere una trasposizione alla lettera degli elementi narrativi di questo brano dantesco che 


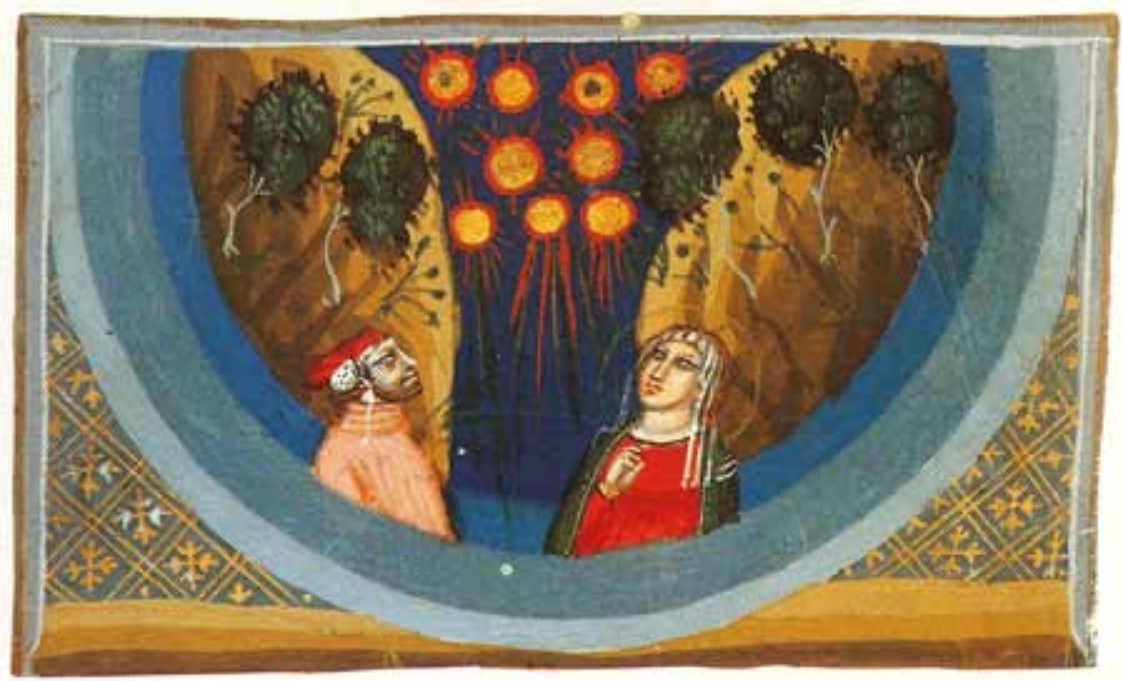

Figura 9. Anonimo, Paradiso XXX, miniatura tratta dal Codex Egerton 943 (prima metà del Trecento), $179 \mathrm{v}$.

${ }^{\odot}$ The British Library Board. (Followed by the shelfmark)(Codex Egerton 943, 179v.).

l'artista ottiene attraverso lo strumento di una simultaneità delle azioni di singole figure angeliche o di gruppi, cosa, questa, che ricorda le immagini in fasi. A margine di questo discorso sia consentito di menzionare il rimando al verso 65 di Par. XXX come prova di una dettagliata trasposizione del brano dantesco: all'indicazione del luogo «ne' fiori» corrisponde l'immersione delle figure angeliche con il busto e a testa in giù nei fiori.

Il disegno botticelliano si può misurare anche con i precetti di un Leon Battista Alberti, secondo cui l'artista deve essere in grado di evocare le attività spirituali attraverso la posizione e l'espressione delle figure ${ }^{43}$. La dimensione mentale del testo trova una molteplice corrispondenza sia nei protagonisti sia nelle figure angeliche. I movimenti di queste ultime rappresentano i sentimenti della voglia, del desiderio, della gioia, della totale dedizione mistica, di un'indomabile vitalità. La mistica del Paradiso, per la cui simbologia in Dante svolgono un ruolo straordinario le gemme con la loro metafisica della luce (la qual cosa caratterizza la metaforicità del testo e il suo carattere di simulacro) in Botticelli, che trasforma le gemme in creature angeliche, non viene rappresentata con la stessa sublimità del testo dantesco. Botticelli, insomma, non raggiunge lo stesso grado di sublime di Dante. Sebbene il pittore, maneggiando con virtuosismo la matita, sappia esprimere una preziosità nelle sue forme,

43. Cfr. Jacqueline Risset, Da Botticelli a Dante. Nascita di Beatrice, in Dante Alighieri, La Divina Commedia. Illustrazioni Sandro Botticelli, a cura di Diane de Selliers, Firenze: Le Lettere, 2008 (1996 $)$, pp. 11-25: 20. 
questa forma di rappresentazione non è sufficiente a rendere la preziosità nel doppio senso in cui in Dante viene prodotta attraverso le gemme, la quale è da descrivere tanto allegoricamente quanto retoricamente.

Osservando da vicino i disegni di entrambi i protagonisti, in base ai movimenti espressivi, alla direzione in cui sono rivolte le parti del corpo e a come sono stati disegnati i loro vestiti, possiamo inequivocabilmente dedurne i processi mentali e le intenzioni gestuali. Allontanandosi dal testo poetico, che mettendo in bocca a Beatrice ultime raccomandazioni per il figlio terreno evita una chiara parola di commiato, Botticelli rappresenta in modo quasi impercettibile un saluto di addio che Beatrice rivolge a Dante, un commiato che il viaggiatore nell'aldilà comprenderà solo nel Canto XXXI. Nella direzione accentuata, nonostante la copertura del vestito, delle gambe di Beatrice, che arrivano a toccare la superficie della "mirabil primavera», secondo il metodo compositivo in fasi adottato da Botticelli, si potrebbe forse vedere un indizio oppure un'anticipazione del suo ritorno nella Rosa dei Beati, la quale pochi versi dopo, originandosi dal fiume di luce, si aprirà allargandosi in un cerchio più grande. Gli occhi di Dante invece parlano un'altra lingua. Lo sguardo fisso indirizzato alla "mirabil primavera» come «umbrifero prefazio" appare rivolto verso l'interno e non veramente capace di una percezione esterna, con la qual cosa l'artista Botticelli finge di rappresentare l'esperienza mistica della grande visione. I movimenti dell'anima del pellegrino in cerca della verità sono suggestivamente accennati attraverso la posizione del corpo e il linguaggio degli occhi della figura disegnata. La forza espressiva della figura di Beatrice, unitamente alla dinamica delle "faville vive» sotto forma di angeli velati di fiammelle, esercita un fascino sull'osservatore attraverso un evidente accumulo di energia. A ciò corrisponde la percezione intuitiva di un'illusione provocata dal disegnatore, ossia l'immaginazione di una metaforicità sovrannaturale che nella sua interezza si oppone alla legge della gravità terrestre. Da questo punto di vista, grazie alla sottile ricezione della poesia dantesca e nonostante alcune differenze, il disegno botticelliano per il Canto XXX del Paradiso offre una trasposizione ben riuscita di una dimensione mistica della visione interiorizzata della trascendenza.

Le esperienze-limite del poeta, che negli ultimi Canti del Paradiso lo portano sempre più a lamentare la mancanza di parole adeguate, non hanno certo mancato di influenzare anche Botticelli. A quanto pare, l'artista si è arreso già con il foglio per il Canto XXX del Paradiso, ultimo disegno eseguito per il ciclo. A ciò ci induce a credere la scelta del soggetto, la limitazione alle 'ombre' ("prefazi») e così lo 'spettacolo' dinanzi alla 'cortina' («dipinte»). Il word-painting della Rosa dei Beati, che appare nello stesso Canto nella sua paradossale metaforicità surrealistica, non è immaginabile, come ha dimostrato Andreas Kablitz ${ }^{44}$, dal momento che mette fuori gioco le leggi naturali («ché dove Dio sanza mezzo governa, / la legge natural nulla rileva», Par. XXX, 122-123), 
ponendo l'artista rinascimentale dinanzi a problemi irrisolvibili. Da questo punto di vista si può ritenere giustificatamente, o almeno comprensibilmente, che Botticelli si sia fermato su questo limite rinunciando in definitiva all'esecuzione dei fogli per i Canti dal XXXI al XXXIII ${ }^{45}$.

\section{Bibliografia}

\subsection{Testi di riferimento}

Alighieri, Dante, La Vita Nuova di Dante Alighieri, a cura di Michele Barbi, Edizione critica, Firenze: Bemporad \& Figlio, $1932\left(1907^{1}\right)$.

- Convivio, Edizione Nazionale delle Opere di Dante, a cura di Franca Brambilla. Firenze: Le Lettere, 1995.

- La Divina Commedia, a cura di Natalino Sapegno, 3 voll., Firenze: La Nuova Italia, $1982^{14}\left(1957^{1}\right)$.

- La Commedia secondo l'antica vulgata, a cura di Giorgio Petrocchi, Firenze: Le Lettere, 1994 (1966-1967¹, Milano: A. Mondadori).

- Commedia con il commento di Anna Maria Chiavacci Leonardi, Bologna: Zanichelli, 2001.

- Die Göttliche Komödie, übersetzt von Hermann Gmelin, Stuttgart: Reclam, 1980.

- Die göttliche Komödie, erläutert von Ferdinand BARTH aufgrund der Übersetzung von Walter Naumann, Darmstadt: Wissenschaftliche Buchgesellschaft, 2004.

Guinizzelli, Guido, Rime, in Poeti del Duecento, a cura di Gianfranco Contini, Milano-Napoli: Riccardo Ricciardo Editore, 1960, tomo II, pp. 450-485.

Latini, Brunetto, Tresor, a cura di Pietro G. Beltrami, Paolo Squillacioti, Plinio Torri, Sergio Vatteroni, Torino: Einaudi, 2007.

Magnus, Albertus, De mineralibus, Basel: Birkhäuser Verlag, 1983.

Marbodo di Rennes, Lapidari, La magia delle pietre preziose, a cura di Bruno Basile, Roma: Carocci, 2006.

\subsection{Letteratura critica}

Bashir-Hecht, Herma, «Die Paradieslandschaft bei Dante und Botticelli», Die Kunst, 97, 1985, pp. 1031-1034.

Batard, Yvonne, Les dessins de Sandro Botticelli pour la Divine Comédie, Paris: Perrin, 1952 ("Collection Jeu savant», 2).

Bellini, Paolo, «Le due serie di disegni del Botticelli per la Commedia», in GizzI, Botticelli e Dante..., pp. 41-50.

Berenson, Bernard, I disegni dei pittori fiorentini, 3 voll., Milano: Electa, 1961. (Prima edizione: The Drawings of the Florentine Painters, 2 voll., London 1903; edizione ampliata: 3 voll., Chicago 1938; per l'edizione italiana del 1961 il testo fu ulteriormente modificato).

Bertini, Aldo, I grandi maestri del disegno. Botticelli, Milano: Aldo Martello, 1953 (edizione inglese: Drawings by Botticelli, The Great Masters of Drawing, New York: Dover, 1968).

Bertolucci Pizzorusso, Valeria, «Gli smeraldi di Beatrice» (1969), in ID, Morfologie del testo medievale, Bologna: Il Mulino, 1989, pp. 199-207.

45. Cfr. Schulze Altcappenberg, Inferno. Purgatorio. Paradiso, op. cit., p. 288. 
Binni, Walter, "Il canto XXX del Paradiso» (1965), in Lectura Dantis Scaligera, vol. III: Paradiso, Firenze: Le Monnier, 1968, pp. 1061-1092.

Bologna, Corrado, Il ritorno di Beatrice. Simmetrie dantesche tra Vita Nova, "petrose» $e$ Commedia, Roma: Salerno Editore, 1998.

- Il canto XXXI del Purgatorio, in Lectura Dantis Turicensis, a cura di Georges Güntert, Michelangelo Picone, vol. II, Purgatorio, Firenze: Franco Cesati, 2002, pp. 473-493.

- «Il canto XXX del Paradiso», in Lectura Dantis Turicensis, a cura di Georges GüNTERT, Michelangelo Picone, vol. III, Paradiso, Firenze: Franco Cesati, 2002, pp. 457-472.

Bredekamp, Horst, Die Medici, Sixtus IV. und Savonarola: Botticellis Konflikte, in Schulze Altcappenberg, Sandro Botticelli..., pp. 292-297.

Chiarini, Eugenio (a cura di), "Il canto XXX del Purgatorio», in Lectura Dantis Scaligera, Firenze: Le Monnier, 1965, pp. 1103-1138.

Chiavacci Leonardi, Anna Maria, "Il canto XXX del Paradiso», Paragone / Letteratura, 26, 1975, Nr. 308, pp. 3-34.

Clark, Kenneth, The Drawings by Sandro Botticelli for Dante's Divine Comedy after the Originals in the Berlin Museums and the Vatican, London: Thames and Hudson, 1976.

Crivelli, F., «Le pietre nobili nelle opere di Dante», Giornale dantesco, 48, 1920, pp. 49-66.

Dombrowski, Damian, «Beobachtungen zu Botticellis Dante-Illustrationen», Deutsches Dante Jahrbuch, 71, 1996, pp. 45-75.

- Botticelli und die Konstruktion des Geistes. Die Dante-Zeichnungen und die Grenzen der Kulturgeschichte, in Schulze Altcappenberg, Sandro Botticelli..., pp. 298-305.

Donati, Lamberto, Il Botticelli e le prime illustrazioni della Divina Commedia, Firenze: Olschki, 1962.

Dronke, Peter, «Symbolism and Structure in Paradiso XXX», Romance Philology, 43, 1989, pp. 29-48.

Gizzi, Corrado (a cura di), Botticelli e Dante, Milano: Electa, 1990.

Hollander, Robert, "Paradiso XXX», Studi Danteschi, 40, 1988, pp. 1-33.

Hughes Gillerman, Dorothy, «Trecento Illustrators of the Divina Commedia», Annual Report of the Dante Society, 1959, pp. 1-40.

Kablitz, Andreas, Poesie der Wissenschaft: Dantes Kosmologie, in Herbert Jaumann, Jürgen Klein, Bettina Rommel, Gregor Vogt-Spira (a cura di), Domänen der Literaturwissenschaft, Tübingen: Stauffenburg, 2001, pp. 233-252.

KorZeniewski, Uta, Karfunkelstein und Rosenquarz. Mythos und Symbolik edler Steine, Ostfildern: Jan Thorbecke Verlag, 2005.

Levavasseur, A., "Les pierres précieuses dans la Divine Comédie», Revue des Études italiennes, 4, 1957, pp. 31-100.

Lipinsky, Angelo, "La simbologia delle gemme nella Divina Commedia e le sue fonti letterarie», in Atti del $1^{\circ}$ congresso nazionale di studi danteschi, Firenze: Olschki, 1962, pp. 127-158.

Lippmann, Friedrich, Zeichnungen von Sandro Botticelli zu Dantes Göttlicher Komödie nach den Originalen im K. Kupferstichkabinett zu Berlin, 2 voll., Faksimile der Illustrationen und erklärende Beschreibung, II, Berlin: Grote, $1921^{2}\left(1887^{1}\right)$.

- (a cura di), Zeichnungen von Sandro Botticelli zu Dantes Göttlicher Komödie. Verkleinerte Nachbildungen der Originale im Kupferstichkabinett zu Berlin und in der Bibliothek des Vatikans, mit einer Einleitung und der Erklärung der Darstellungen, Berlin: Grote, $1921^{2}$. 
Loos, Erich, «Illustrationen zu Dantes Commedia als Beiträge zur Deutungsgeschichte», Deutsches Dante-Jahrbuch, 60, 1985, pp. 153-171.

Oltrogge, Doris, Fuchs, Robert, Hahn, Oliver, Finito und Nonfinito. Zur Technik von Zeichnung und Malerei in Botticellis Divina Commedia, in Schulze Altcappenberg, Sandro Botticelli... pp. 334-341.

Parronchi, Alessandro, "Le illustrazioni del Botticelli per la Commedia e il progetto di un 'Pantheon' fiorentino", in GizzI, Botticelli e Dante..., pp. 77-80.

Risset, Jacqueline, «Da Botticelli a Dante. Nascita di Beatrice», in Dante Alighieri, La Divina Commedia. Illustrazioni Sandro Botticelli, a cura di Diane DE Selliers, Firenze: Le Lettere, $2008\left(1996^{1}\right)$, pp. 11-25.

Salsano, Fernando, Il canto XXX del Paradiso, in Nuove letture dantesche, VII, Firenze: Le Monnier, 1974, pp. 215-234.

Savj-Lopez, Paolo, "Paradiso - Canto XXX", in Letture dantesche, III: Paradiso, a cura di G. Getto, Firenze: Sansoni, 1964, pp. 625-638.

Schulze Altcappenberg, Hein-Th. (a cura di), Sandro Botticelli. Der Bilderzyklus zu Dantes Göttlicher Komödie. Mit einer repräsentativen Auswabl von Zeichnungen Botticellis und illuminierten Commedia-Handschriften der Renaissance, OstfildernRuit, Hatje Cantz und London, Royal Academy of Arts, 2000.

- 'per essere persona sofistica'. Botticellis Bilderzyklus zur Göttlichen Komödie, in ID. (a cura di), Sandro Botticelli..., op. cit., pp. 14-35.

- Der Bilderzyklus von Sandro Botticelli. Inferno. Purgatorio. Paradiso, in ID. (a cura di), Sandro Botticelli..., op. cit., pp. 37-290.

Scott, J.A., Paradiso XXX, in Dante Commentaries. Eight Studies of the Divine Comedy, a cura di D. Nolan, Dublin-Totowa: Irish Academic Press, 1977, pp. 159-180.

Sollers, Philippe, Dante et la traversée de l'écriture (1965), in ID., Logiques, Paris: Seuil, 1968, pp. 44-77.

Studer, Paul, Evans, Joan, Anglo-Norman Lapidaries, Paris: Champion, 1924.

Supino, Igino Benvenuto, I disegni per la Divina Commedia di Dante, Bologna, 1921.

Tobler, Adolf, Lommatzsch, Erhard, Altfranzösisches Wörterbuch, Berlin, Weidmann u. Wiesbaden: Steiner, 1915.

Vallone, Aldo, "Il canto XXX del Paradiso e la 'luce intellettuale'», L'Alighieri, 27, 1986, Nr. 2, pp. 63-78.

Venturi, Adolfo, Il Botticelli interprete di Dante, Firenze: Le Monnier, 1921.

Volkmann, Ludwig, Iconografia dantesca. Die bildlichen Darstellungen zur Göttlichen Komödie, Leipzig: Breitkopf \& Härtel, 1897.

Witke, Edward Charles, "The River of Light in the Anticlaudianus and the Divina Commedia», Comparative Literature, 11, 1959, pp. 144-156. 